\title{
Lesions of the Hippocampal Formation But Not Lesions of the Fornix or the Mammillary Nuclei Produce Long-Lasting Memory Impairment in Monkeys
}

\author{
Stuart Zola-Morgan, ${ }^{1}$ Larry R. Squire, ${ }^{1}$ and David G. Amaral ${ }^{2}$ \\ 'Veterans Administration Medical Center, San Diego, California 92161, and Department of Psychiatry, University of \\ California, San Diego, La Jolla, California 92093, and ${ }^{2}$ The Salk Institute, San Diego, California 92138
}

\begin{abstract}
A group of tasks sensitlve to human amnesia were used to characterize the severity and duration of memory impairment in monkeys following bilateral damage to the hippocampal tormation, fornix, or mammillary nuclei. Monkeys with hippocampal formation lesions (which included the hippocampus proper, dentate gyrus, subiculum, posterior entorhinal cortex, and much of the parahippocampal gyrus) exhibited a substantial and lasting memory impairment. Monkeys with fornix transection or bilateral damage to the mammillary nuclei were impaired on the first task administered after surgery (delayed nonmatching to sample). However, they performed all the other tasks normally and were unimpaired when the delayed nonmatching to sample task was re-administered 18 months after surgery. The findings are consistent with reports that damage limited to the human hippocampus can produce a clinically significant and permanent amnesia. Because fornix transection or mammillary lesions produced only transient memory impairment, it seems unlikely that similar damage in humans can cause a severe or permanent amnesia.
\end{abstract}

Bilateral damage to the medial temporal lobe or to the midline diencephalic region of the human brain has been known for nearly a century to cause a profound amnesic syndrome (Gudden, 1896; von Bechterev, 1900). Considerable uncertainty remains, however, about which specific structures and connections within these regions must be damaged (for reviews, see Mair et al., 1979; Damasio, 1984; Squire, 1987). One traditional idea is that amnesia results when damage occurs within the socalled Papez (1937) circuit, which includes the hippocampal formation, fornix, and mammillary (see Appendix) nuclei (Barbizet, 1970; Brierley, 1977).

Recent findings from a carefully studied patient (R.B.) show that amnesia can result from bilateral damage limited to the hippocampus (Zola-Morgan et al., 1986). The effects on human memory of selective damage to the fornix or mammillary nuclei are unclear at this time. While impaired memory has been re-

\footnotetext{
Received May 23, 1988; revised July 20, 1988; accepted July 22, 1988.

Supported by the Medical Research Service of the Veterans Administration, NIH Grants NS19063, NS16980, and MH41479, NSF Grant BSN85-12349, and The Office of Naval Research. We thank Drs. Kasden, Jacobsen, and Holmes for instruction in the surgical approach to the mammillary nuclei; Ellen Bulder, Carol Micheletti, Jasmine Allen, and Jerri Milana for behavioral testing; and Cecelia LeClair, Michael Mesches, and Janet Weber for histological assistance.

Correspondence should be addressed to Stuart Zola-Morgan, Veterans Administration Medical Center, $3350 \mathrm{La} J o l l a$ Village Drive, San Diego, CA 92161.

Copyright (C) 1989 Society for Neuroscience 0270-6474/89/030898-16\$02.00/0
}

ported following bilateral fornix damage (Hassler and Reichert, 1957; Sweet et al., 1959; Heilman and Seipert, 1977), the assessment of memory function in these cases was often based on anecdotal reports, and in no case has comprehensive neuropsychological and neuropathological analysis been conducted. Several cases have also been reported in which memory was apparently intact following fornix damage (Woolsey and Nelson, 1975; see Garcia-Bengochea and Friedman, 1987, for a review), although these reports also provided insufficient neuropsychological and/or neuropathological data.

The mammillary nuclei were initially linked to memory function because they are consistently damaged in alcoholic Korsakoff's syndrome (Barbizet, 1970; Brierley, 1977). Yet, in this disorder the mammillary nuclei are not the only site of damage (Mair et al., 1979). Damage to the mediodorsal nucleus of the thalamus, for example, has been suggested to be responsible for the amnesia of Korsakoff's syndrome, either alone (Victor et al., 1971) or in combination with damage to the mammillary nuclei (Markowitsch, 1982; Butters, 1984).

One promising approach to understanding the anatomy of amnesia is afforded by the recent development of an animal model of human amnesia in the monkey (Mishkin, 1982; Squire and Zola-Morgan, 1983; Mahut and Moss, 1984). The model has relied especially on the delayed nonmatching to sample task, an object-recognition memory task that is sensitive to human amnesia (Squire et al., 1988). Monkeys with bilateral lesions of the hippocampal formation are impaired on this object-recognition task (Mishkin, 1978; Mahut et al., 1982; Murray and Mishkin, 1984; Zola-Morgan and Squire, 1986).

Other studies have evaluated the performance of monkeys on the delayed nonmatching to sample task following lesions of either the mammillary nuclei or the fornix. In one study, monkeys with lesions of the mammillary nuclei were only mildly impaired on the delayed nonmatching to sample task (Aggleton and Mishkin, 1985) and less impaired than monkeys with lesions of the anterior mediodorsal nucleus (Aggleton and Mishkin, 1983a, b). In 2 other studies, monkeys with fornix transection performed as well as controls on the delayed nonmatching to sample task (Mahut et al., 1982) or were only mildly impaired (Bachevalier et al., 1985). In the first study, the monkeys were tested 5 years after surgery, while in the second study, monkeys were trained preoperatively and then tested after surgery.

Several issues remain to be clarified concerning the role of the fornix and mammillary nuclei in memory function. The first issue concerns the severity of memory impairment that results from lesions of each structure. In 2 previous studies, monkeys 
with fornix transection (Bachevalier et al., 1985) or mammillary nuclei lesions (Aggleton and Mishkin, 1985) exhibited at most a mild memory impairment. Monkeys in these studies were trained preoperatively and then tested postoperatively. In other studies of monkeys with hippocampal lesions, performance was also mildly impaired when preoperative training was given (Mishkin, 1978; Murray and Mishkin, 1984). Based on these findings, one might suppose that similar effects on memory should be produced by damage to the hippocampal formation, fornix, or mammillary nuclei. However, performance was substantially impaired following hippocampal formation lesions when training began postoperatively (Mahut et al., 1982; Mahut and Moss, 1984; Zola-Morgan and Squire, 1986; see Ringo, 1988, for further discussion). It remains unclear how monkeys with fornix transection or mammillary nuclei lesions would perform on memory tasks if training began postoperatively and how these 2 groups would perform in comparison to monkeys with hippocampal lesions.

A second issue concerns the nature of the memory impairment exhibited by operated monkeys. Whereas the development of a nonhuman primate model of human amnesia has depended heavily on the delayed nonmatching to sample task, human amnesia affects performance on a variety of different tasks. Recently, monkeys with large bilateral medial temporal lobe lesions were found to be impaired on several different tasks that human amnesic patients fail (Zola-Morgan and Squire, 1985). It would be useful to compare directly the effects of hippocampal lesions, fornix transection, and mammillary nuclei lesions on these same tasks. Information from multiple tasks makes it easier to appreciate both the severity and the scope of the impairment.

A third issue concerns whether or not the memory impairment produced by a lesion is enduring. If damage to a particular brain structure is proposed as a cause of the permanent memory impairment observed in human amnesia, the memory deficit should be long-lasting. In previous studies of monkeys with medial temporal lobe lesions, the deficit was found to persist for at least 1.5 years after surgery (Zola-Morgan and Squire, 1985). The long-term effects of selective hippocampal and diencephalic lesions on memory have not been systematically examined in the monkey.

The present study assessed memory performance in 4 separate groups of monkeys: a normal control group, a group with bilateral lesions of the hippocampal formation, a group with bilateral fornix transection, and a group with bilateral lesions of the mammillary nuclei. In each group, all training and testing began after surgery. In addition to the delayed nonmatching to sample task, we assessed performance on other tests of memory that vary widely in their behavioral requirements and that are sensitive to human amnesia (Squire et al., 1988). We also assessed performance on 2 skill-like tasks that are analogous to tasks that amnesic patients can perform well (Zola-Morgan and Squire, 1984). Testing occurred during the period from 2 months to about 1.5 years after surgery.

\section{Materials and Methods}

Subjects

Seventeen cynomolgus monkeys (Macaca fascicularis) were used, all weighing between 3.7 and $4.5 \mathrm{~kg}$ at the beginning of the study. Based on weight-and-age tables (Szabo and Cowan, 1984; Hartley et al., 1984), these monkeys were estimated to be $4-5$ years old. Three female monkeys received bilateral lesions of the hippocampal formation $\left(\mathrm{H}^{+}\right)$. Some behavioral data for these monkeys were reported previously (tests 1 and
2: Zola-Morgan and Squire, 1986). Six monkeys ( 3 males and 3 females) received bilateral lesions of the fornix $(F X)$. A seventh monkey intended for this operated group did not sustain bilateral damage to the fornix. Histological evaluation revealed unilateral damage to the fornix and a small unilateral lesion of the septum. This animal performed normally on all the tasks and was not included in the data analysis. Histological material from this animal is presented in Figures 2 and 3 to show the normal appearance of the fornix in thionin and AChE preparations.

Two male monkeys received bilateral lesions of the mammillary nuclei (MN). A third monkey, intended for this operated group, sustained only a small unilateral lesion to the mammillary nuclei. A fourth monkey sustained unilateral hypothalamic damage anterior to the mammillary nuclei, which transected the fornix. While there was no direct damage to the mammillary nuclei, there was substantial unilateral shrinkage of the medial mammillary nucleus. A photomicrograph of the normal and shrunken medial mammillary nuclei from this animal is shown in Figure 4. The scores for these 2 animals were not included in the data analysis. The 3 remaining monkeys ( 2 males and 1 female) comprised an unoperated, normal control group (N). Behavioral data for these monkeys were reported previously (tests 1 and 2: Zola-Morgan et al., 1982; tests 3-6: Zola-Morgan and Squire, 1985; test 7: Zola-Morgan and Squire, 1984). Monkeys were maintained on a daily ration of Purina Monkey Chow, supplemented with fruit and chewable vitamin $\mathrm{C}$ tablets (250 $\mathrm{mg}$ ).

Although the groups were not all tested contemporaneously, animals werc given the same scquence of tests (sec bclow). In addition, all animals were tested in the same facility, using the same apparatus and test protocols. A research protocol describing all aspects of the present study that related to the use of animals (care and maintenance, surgery, behavioral testing, and euthanasia) was approved by the Animal Research Committees of the V.A. Medical Center, San Diego, and the University of California, San Diego.

\section{Surgery}

All surgery was performed under sodium pentobarbital anesthesia (30 $\mathrm{mg} / \mathrm{kg}$ ). The surgical procedure used for making the hippocampal lesions has been described previously (Zola-Morgan and Squire, 1986). The hippocampus on each side was approached by elevating the temporal lobe and entering the brain just medial to the occipitotemporal sulcus and caudal to the entorhinal cortex. The hippocampus, dentate gyrus, and subicular complex were removed. The removal also included much of the parahippocampal gyrus (areas TF and TH of von Bonin and Bailey, 1947) and approximately the posterior half of the entorhinal cortex. The lateral ventricle served as an identifiable dorsal boundary along the entire length of the removal.

Lesions of the fornix were made in 2 monkeys by a direct, neurosurgical approach (Mahut, 1972) and in 4 monkeys with a stereotaxic procedure. For the neurosurgical approach, the monkey's head was placed in a stereotaxic instrument (David Kopf, model 712). Following a large dorsal craniotomy, a unilateral dural flap was made, and the medial aspect of the left hemisphere was gently retracted. Under direct vision, a 5-10 mm longitudinal incision of the corpus callosum was made that exposed the frontal horn of the right lateral ventricle. The columns of the fornix were identified and then raised and sectioned by means of a small, sharpened hook at or just behind the foramen of Monro.

For the stereotaxic approach, a radio frequency lesion maker (Grass, model LM-2) was used in combination with a specially designed electrode, $0.5 \mathrm{~mm}$ in diameter, with a tapered tip exposed for $2 \mathrm{~mm}$. Stereotaxic coordinates for placement of the probe into the amygdala were determined from the atlas of Szabo and Cowan (1984). The mediallateral zero-point was determined by thinning the skull until the sagittal sinus could be viewed. The medial-lateral zero-point was taken as the middle of the width of the sinus. Two small craniotomies were then made, one on each side of the midline, extending for $10 \mathrm{~mm}$ in the anterior-posterior (AP) plane and $5 \mathrm{~mm}$ in the medial-lateral plane. A dural flap was made, and the electrode was moved and lowered to the predetermined coordinates. Three separate lesions of the fornix were made on each side of the brain (at AP levels A11.0, A9.0, and A7.0). For each lesion a current of approximately $100 \mathrm{~mA}$ was passed through the electrode for $15 \mathrm{sec}$

For the mammillary nuclei lesions, a direct surgical approach was used similar to that described by Holmes et al. (1983). The skull and dura over the left temporal lobe were opened to gain access to the left and right mammillary nuclei. The monkey's head was held in a specially 
designed head-holder that allowed unobstructed access to the temporal and ventral portions of the cranial cavity. After exposing the tempora muscle and bone on one side, the zygomatic arch was removed to facilitate entrance to the temporal fossa, and the dura was opened exposing the lateral surface of the temporal lobe. The animal was then placed in a supine position that allowed the brain to compress gently against the top of the skull, thereby providing access between the base of the skull and the base of the brain. When the temporal lobe was then gently retracted, the base of the brain hecame accessible across the midline. The left third cranial nerve was sectioned just rostral to the cerebral peduncle. Finally, the arachnoid surrounding the pituitary stalk and adjacent regions on each side was carefully removed, exposing the mammillary nuclei. Under direct vision, a cauterizing electrode was inserted several times into the mammillary nuclei on each side of the midline, and a current of $100 \mathrm{~mA}$ was passed through the electrode for 10-15 sec.

\section{Behavioral testing}

Monkeys were allowed 6-8 weeks of recovery prior to the start of behavioral testing. All testing was carried out in a Wisconsin General Test Apparatus (Harlow and Bromer, 1938). During 4-6 sessions of pretraining, monkeys learned to obtain food by displacing objects that covered any of 3 food wells located on a stimulus tray in front of the testing chamber. Seven different tasks (described in detail in Zola-Morgan and Squire, 1984, 1985) were administered to all monkeys in the order listed below.

1. Trial-unique delayed nonmatching to sample. Monkeys first displaced an object covcring the ccntral food wcll to obtain a raisin reward, following which an opaque door was lowered to block the monkey's view of the food wells. After $8 \mathrm{sec}$, monkeys saw 2 objects, the original object and a new one covering the 2 lateral food wells, and their task was to displace the new object to obtain the raisin. The position of the correct object (left or right) varied on each trial according to a pseudorandom schedule (Gellerman, 1933). Twenty such trials were presented daily with an intertrial interval of $20 \mathrm{sec}$. Each trial used a new pair of objects, selected randomly from a collection of more than 300 junk objects. After reaching the learning criterion of 90 correct choices in 100 trials, monkeys were tested with successively longer delays of 15 $\mathrm{sec}, 60 \mathrm{sec}$, and $10 \mathrm{~min}$ between the sample and choice trials. One hundred trials were given at the 15 and $60 \mathrm{sec}$ delays; 50 trials were given at the $10 \mathrm{~min}$ delay. Twenty trials were given daily for the 15 and $60 \mathrm{scc}$ dclays, and 5 trials were given daily for the 10 min delay.

2. Pattern discrimination. Monkeys were next tested on 2 pattern discrimination tasks. For these tasks, color, size, and 3-dimensional shape information are not available. In the first task, monkeys learned to discriminate a plus sign from a square, and in the second task they learned to discriminate an $\mathrm{N}$ from a W. A correct choice uncovered a raisin reward, and an incorrect choice uncovered an empty food well. The position of the correct plaque (over the left or right lateral food wells) was determined by a Gellerman sequence. Training continued until animals achieved a learning criterion of $90 \%$ correct performance or better on 2 consecutive days. Twenty trials per day were administered for the first task, and 30 trials per day were administered for the second task.

3. Delayed retention of object discriminations. Monkeys learned 4 scparatc 2-choicc object discrimination tasks. Each discrimination pair consisted of distinctive objects that could be learned by normal monkeys in a single session. Each discrimination task was administered for 2 daily sessions of 20 trials each, with an intertrial interval of $15 \mathrm{sec}$ Following a $2 \mathrm{~d}$ delay, an additional session of 20 trials was given. The correct stimulus object appeared over the left or right food wells in a Gellerman sequence, and a raisin reward was always concealed under the correct object. Five to $7 \mathrm{~d}$ intervened between each of the 4 discrimination problems.

4. Concurrent discrimination learning. Eight pairs of junk objects were used. The pairs were presented in an intermingled fashion during each testing session so that all 8 discriminations had to be learned simultaneously. Specifically, on each trial one pair of objects was present over the lateral food wells, and during the course of each daily testing session of 40 trials, every pair was presented 5 times. The intertrial interval was $15 \mathrm{sec}$. The same object of a pair was always correct each time it was presented. The position of the correct object (left or right) was determined by a Gellerman sequence, and a raisin reward was always concealed under the correct object. Testing continued until a learning criterion of 39 correct responses in 40 consecutive trials was achieved during one test session.
5. Delayed response with and without distraction. Testing proceeded in 2 phases, basic training and then testing with delays. Basic training: With the opaque door in the raised position, 1 of the 2 lateral food wells was baited with a raisin reward while the monkey watched. Both food wells were then covered by identical square black plaques. The opaque screen was then lowered between the monkey and the food wells. After a delay of approximately $8 \mathrm{sec}$, the opaque screen was raised, and the monkey was allowed to choose between the 2 covered food wells. Monkeys were tested for 20 trials per day to a learning criterion of 90 correct responses in 100 consecutive trials. The intertrial interval was 20 sec. Testing on delays (with and without distraction): Monkeys were tested with short $(15 \mathrm{sec})$ and long $(30 \mathrm{sec})$ delays, beginning on the day after criterion was reached on the basic task. Five conditions ( $8 \mathrm{sec}-\mathrm{no}$ distraction; 15 sec-no distraction; 15 sec-distraction; 30 sec-no distraction; 30 sec-distraction) were presented in daily sessions of 25 trials. Each condition occurred randomly 5 times a day. In all, 10 daily sessions were administered for a total of 50 trials in each condition. For the 3 no-distraction conditions, testing was the same as in basic training except that the delay could be 8,15 , or $30 \mathrm{sec}$. For the 2 distraction conditions, the plaques and the bait were quickly removed and a distracting event was introduced during the delay interval. The distracting event consisted of the presentation of an irrelevant junk object (once during the $15 \mathrm{sec}$ delays or 3 times during the $30 \mathrm{sec}$ delays), which monkeys could displace to obtain a raisin reward. The same object was used as the distractor throughout testing.

6. Delayed nonmatching to sample with and without distraction. Basic training: This was a re-administration of the basic task (8 sec delay) described in test 1 , above. Testing on delays (with and without distraction): Following completion of training on the basic task, the effects of delay and distraction were investigated in the same way as described above for delayed response. The same short $(15 \mathrm{sec})$ and long $(30 \mathrm{sec})$ delays and distracting task were used but with a different distracting object. The average interval between test 1 and test 6 was 21 months.

In addition, monkeys in the $\mathrm{H}^{+}$group were given the delayed nonmatching to sample task on 2 additional occasions at 42 and 53 months after surgery. On these occasions, the task was given in the identical way that it had been given originally, 2 months after surgery.

7. Lifesaver motor-skill task. This task was adapted from one described by Davis et al. (1956). Monkeys learned to obtain an edible Lifesaver candy by maneuvering it a distance of 4 inches along a metal rod and around a right angle turn for a distance of 1.5 inches. Prior to formal testing, monkeys were first pretrained to retrieve the candy Lifesaver when it was placed directly at the end of the metal rod and could be removed without difficulty. For formal testing, 6 trials a day were given in which the monkey was allowed $30 \mathrm{sec}$ to retrieve the Lifesaver by maneuvering it from the center of the rod, around the bend, and off the end of the rod. If $30 \mathrm{sec}$ elapsed before the monkey obtained the Lifesaver, the opaque door was lowered and the trial was reset with the Lifesaver at the center of the rod. Sessions were given every other day, for a total of 8 sessions. One month after the eighth learning session, monkeys were retested by giving them 2 additional sessions on 2 consecutive days.

\section{Histological evaluation}

At the completion of behavioral testing, animals were deeply anesthetized with Nembutal and euthanized by transcardial perfusion. In most cases, animals were first perfused with a $0.9 \%$ solution of $\mathrm{NaCl}$ followed by at least 2 liters of $4 \%$ paraformaldehyde in $0.1 \mathrm{M}$ phosphate buffer. The brains were then blocked in situ in the coronal plane, removed from the skull, and either placed directly into a cryoprotectant solution of $20 \%$ glycerol in $0.1 \mathrm{M}$ phosphate buffer or placed in fixative for at least a week prior to cryoprotection. In some cases, brains were encapsulated in a $20 \%$ egg albumin solution that was polymerized by adding $1 \%$ glutaraldehyde. Coronal sections $(40-50 \mu \mathrm{m})$ were cut on a freezing microtome, and every fifth section was mounted on slides and stained with thionin. In 3 of the brains prepared with fornix transections, an additional series of sections was stained for the demonstration of AChE.

The series of coronal sections was evaluated microscopically to determine the completeness of the intended lesion and the extent of extrancous damage. Early in the assessment period we noted that the mammillary nuclei were markedly shrunken in animals with damage of the hippocampal formation or fornix. This shrinkage is likely a consequence of deafferentation sustained by the medial mammillary nucleus after the elimination of its input from the subiculum. To estimate the extent of shrinkage, we determined the volume of the medial mammillary nucleus in each animal. For each brain, the outlines of the medial 
mammillary nuclei were traced from all of the sections through the nucleus using a Nikon stereomicroscope and drawing attachment. The area within each outline was then measured using a digitizing tablet linked to a microcomputer, and the volume of the nucleus was calculated. For comparison purposes, the volume of the mammillary nucleus was similarly measured in 4 control animals of like size and weight who were part of a separate neuroanatomic study (Table 1).

\section{Results}

Histological findings

Hippocampal lesions

Two of the three animals with intended hippocampal lesions had complete, bilateral removal of the dentate gyrus, hippocampus, and subicular complex (subiculum, presubiculum, and parasubiculum). The posterior entorhinal cortex and the parahippocampal gyrus were also extensively damaged bilaterally. There was some sparing of the anterior entorhinal cortex in both cases, but cells in layer II, which project to the ablated hippocampal fields, were nearly completely eliminated through retrograde degeneration. The third animal had an incomplete lesion that damaged about $50 \%$ of the hippocampal formation. Damage to the parahippocampal gyrus was also less extensive in this animal than in the 2 others. Additional information about each animal is provided below.

Monkeys $\mathrm{H}^{+} 1$ and $\mathrm{H}^{+} 2$ sustained complete bilateral hippocampal removals (Fig. 1). The rostral third of the entorhinal cortex was intact, but layer II had degenerated (Fig. 1, $A, B$ ). The posterior entorhinal cortex was directly damaged by the lesion (Fig. 1, $C, D$ ), and the lesion extended laterally, more so in $\mathrm{H}^{+} 1$ than in $\mathrm{H}^{+} 2$, to encompass the parahippocampal gyrus bilaterally (Fig. $1 F$ ). In $\mathrm{H}^{+} 1$ the amygdaloid complex was intact (Fig. $1, A, B$ ); in $\mathrm{H}^{+} 2$ there appeared to be slight direct damage of the amygdaloid complex involving the ventral limit of the posterior border of the lateral nucleus. In both animals, the fimbria was shrunken bilaterally and gliotic. In $\mathrm{H}^{+} 1$, the medial mammillary nuclei were approximately $69 \%$ of the volume of control animals, and in $\mathrm{H}^{+} 2$ they were approximately $65 \%$ of the control volume (Table 1 ).

Monkey $\mathrm{H}^{+} 3$ had a smaller lesion involving about half of the hippocampal formation bilaterally. Approximately the anterior $30 \%$ of the hippocampal formation and the posterior $20 \%$ were uninvolved by the ablation. The entorhinal cortex was intact, but there was partial loss of layer II cells. There was also bilateral damage of the parahippocampal gyrus, but this was not as extensive as in the other 2 monkeys. The amygdaloid complex was not involved by the lesion, but at caudal levels the ablation exceeded the dorsal limit of the ventricle and directly damaged the tail of the caudate nucleus bilaterally. The lateral geniculate nucleus was also directly damaged on the left side and was atrophic throughout. The optic radiations appeared to have been damaged on the left side as well. On the right side, the lateral geniculate nucleus was not directly involved in the lesion but also appeared atrophic. The optic radiations on the right side may have been partially damaged. The medial mammillary nuclei were markedly shrunken as in the other cases and had a volume approximately $56 \%$ of control values.

\section{Fornix lesions}

The lesions of the fornix were complete bilaterally in the 2 monkeys prepared by direct surgical approach (FX1 and FX2; Figs. 2, 3) and in 2 of the 4 monkeys who underwent stereotaxic radio frequency ablation of the fimbria (FX3 and FX4). The 2 remaining monkeys in the stereotaxic group (FX5 and FX6) had nearly complete bilateral lesions of the fornix but had slight,

\begin{tabular}{cccc}
\hline \multicolumn{3}{l}{ Table 1. Volume of the medial mammillary nuclei $\left(\mathbf{m m}^{3}\right)$} \\
Animal & Left & Right & Total \\
\hline Control & & & \\
1 & 1.96 & 1.98 & 3.94 \\
2 & 2.02 & 2.06 & 4.08 \\
3 & 2.30 & 2.45 & 4.75 \\
4 & 2.03 & 1.82 & 3.85 \\
Mean & 2.08 & 2.08 & 4.16 \\
$\mathrm{H}^{+}$ & & & \\
1 & 1.44 & 1.41 & 2.85 \\
2 & 1.41 & 1.28 & 2.69 \\
3 & 1.16 & 1.15 & 2.31 \\
Mean & 1.34 & 1.28 & 2.62 \\
FX & & & \\
1 & 1.34 & 1.63 & 2.97 \\
2 & 0.87 & 0.73 & 1.60 \\
3 & 0.81 & 0.83 & 1.64 \\
4 & 1.28 & 1.64 & 2.92 \\
5 & 1.45 & 1.36 & 2.81 \\
6 & 0.84 & 1.03 & 1.87 \\
Mean & 1.10 & 1.20 & 2.30 \\
MN & & & \\
1 & 0.10 & 0.05 & 0.15 \\
2 & 0.00 & 0.03 & 0.03 \\
Mean & 0.05 & 0.04 & 0.09 \\
\hline
\end{tabular}

Left, left medial mammillary nucleus; Right, right medial mammillary nucleus $\mathrm{H}^{+}, \mathrm{FX}, \mathrm{MN}$, monkeys with lesions of the hippocampal formation, fornix, and mammillary nuclei, respectively. Control animals were monkeys from a separate neuroanatomical study.

nearly complete bilateral lesions of the fornix but had slight, unilateral sparing of the lateral tip. For monkeys FX1 and FX2, the completeness of the transections was determined both by analyzing standard thionin-stained sections (Fig. 2) and by studying the distribution of AChE in the fornix at levels proximal and distal to the transection (Fig. 3). Cholinergic fibers originating in the septal complex project to the hippocampal formation via the fornix. In the complete transections, there was a buildup of AChE staining in fibers proximal to the cut (i.e., on the septal side of the transection) and a complete elimination of $\mathrm{AChE}$ staining distal to the transection (Fig. 3).

In monkey FX1 there was little extraneous damage, and the medial mammillary nuclei were shrunken to approximately $71 \%$ of control values. In monkey FX2 there was minor damage to the anterior nuclear complex on the left side. The medial mammillary nuclei in this animal had a volume approximately $38 \%$ of control values.

In monkey FX3, the lesion extended ventral to the fornix and bilaterally damaged a small portion of the caudal pole of the mediodorsal and laterodorsal nuclei. The dorsomedial limit of the pulvinar was damaged on the left side. The stria medullaris and the stria terminalis were also damaged unilaterally. There was minor bilateral damage to the cingulate cortex. The medial mammillary nuclei were shrunken to $39 \%$ of normal values. In monkey FX4, the laterodorsal nucleus was slightly involved on the right side, but there was no damage to the mediodorsal nucleus. There was slight bilateral involvement of the ventral cingulate cortex. The medial mammillary nuclei had a volume approximately $70 \%$ of control values.

In monkeys FX5 and FX6, the fornix on the left side was 

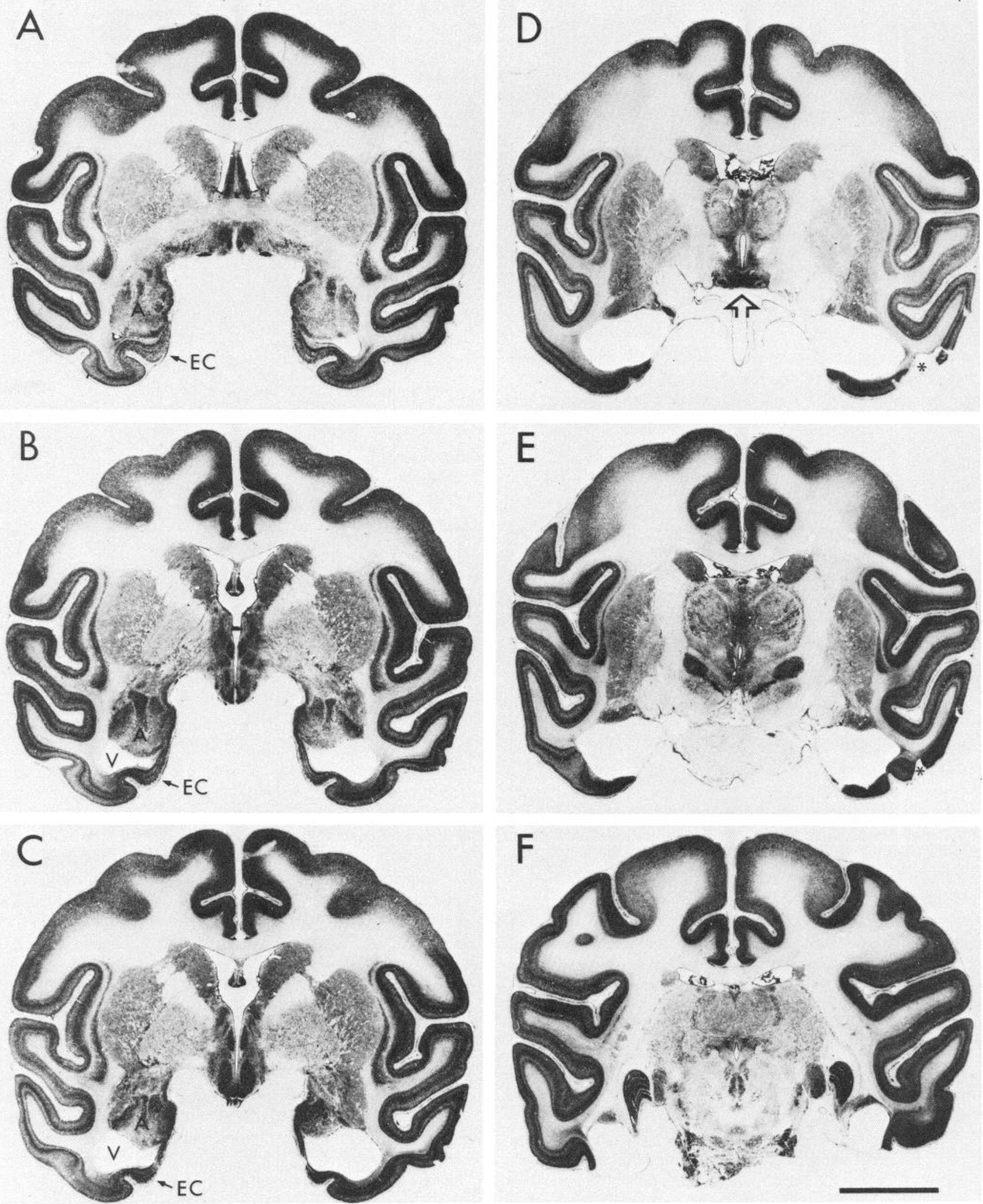

Figure 1. Representative thionin-stained, coronal sections arranged from rostral $(A)$ to caudal $(F)$ through the hippocampal formation of case $\mathrm{H}^{+} 1$. The amygdaloid complex (labeled $A$ in panels $A-C$ ) was not involved in the lesion nor was the rostral half of the entorhinal cortex (labeled $E C$ in panels $A-C$ ). The posterior half of the entorhinal cortex and the full rostrocaudal extent of the other hippocampal fields were completely and bilaterally removed. At caudal levels, the ablation continued laterally to the occipitotemporal sulcus and included much of the parahippocampal fields TH and TF. Asterisks in $D$ and $E$ indicate damage produced in the histological processing. Scale bar, $10 \mathrm{~mm}$. 

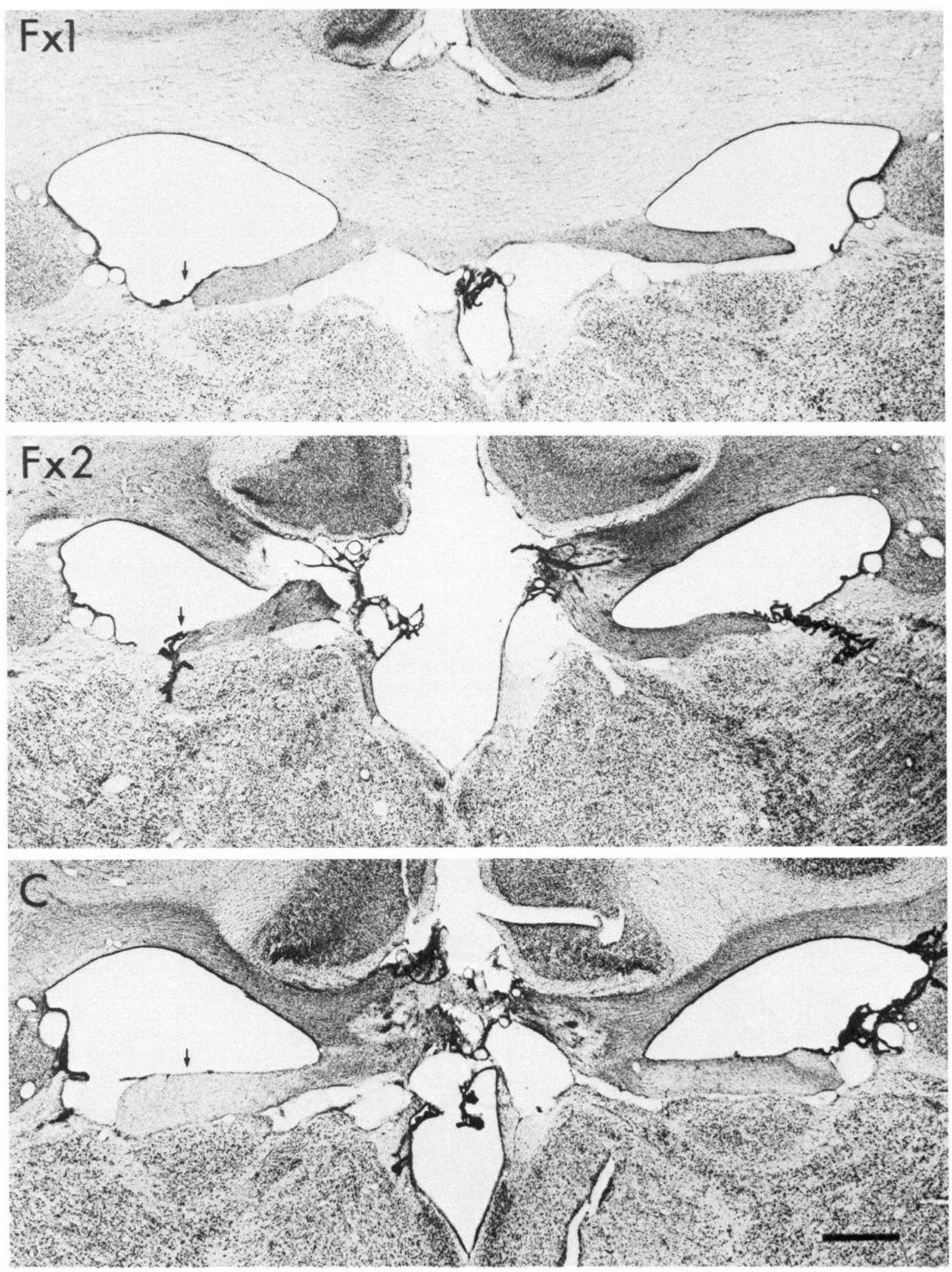

Figure 2. Coronal thionin-stained sections located immediately caudal to the fornix transections in cases $F X 1$ and $F X 2$. A similar level is shown in a third animal $(C)$, in which the lesion unilaterally involved the septal nuclei but did not transect the fornix. Small arrows are placed above the fornix approximately $5 \mathrm{~mm}$ lateral to the midline in each case. Note that there is marked shrinkage and gliosis of the fornix in the top 2 panels. Scale bar, $1 \mathrm{~mm}$. 

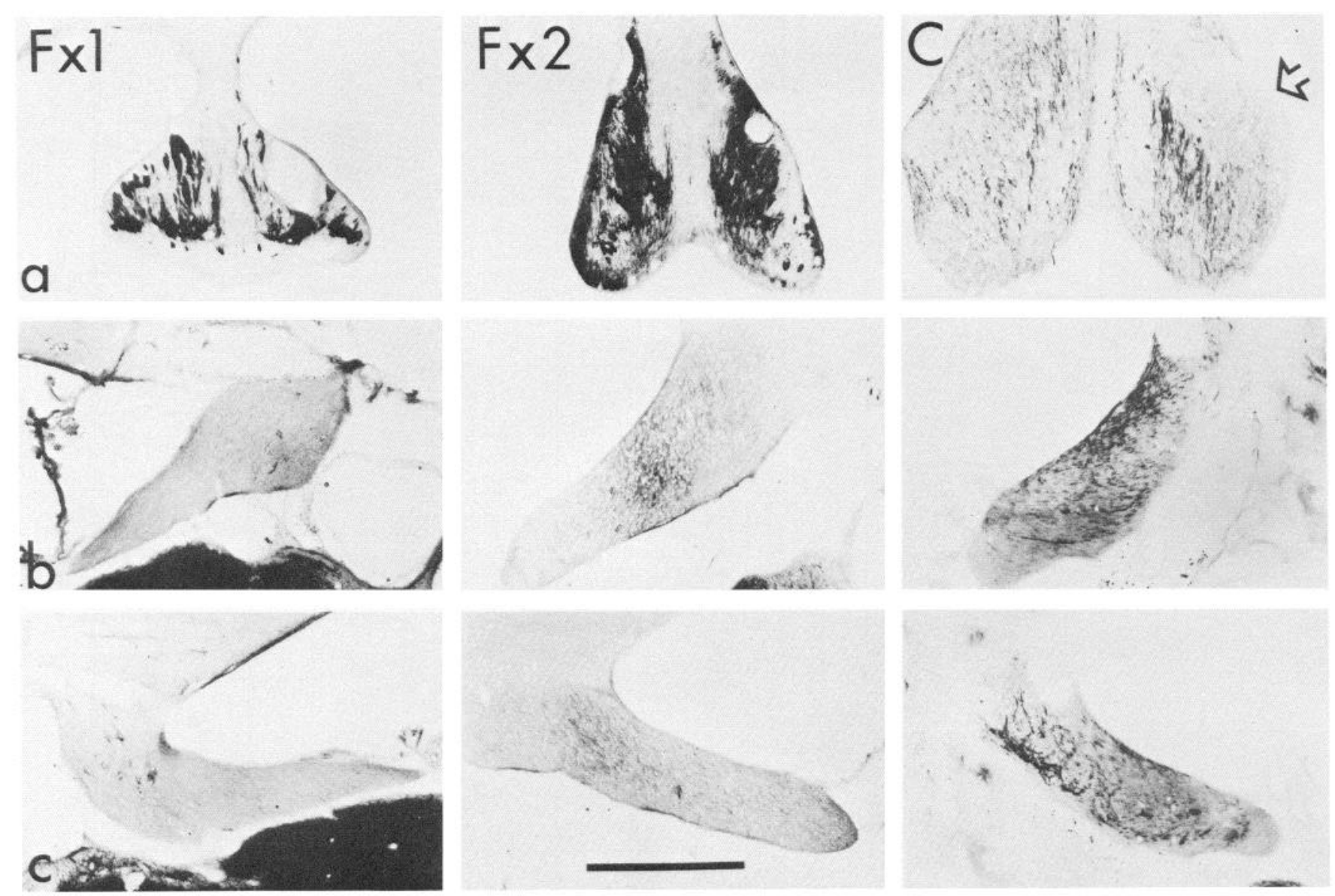

Figure 3. Higher-magnification photomicrographs of acetylcholinesterase preparations of the fornix in cases $F X 1$ and $F X 2$ in which the fornix was completely transected. A third animal $(C)$ is also shown, in which the lesion involved the right side of the septal complex and did not directly damage the fornix. In $a$, the fornix is shown at a level rostral to the transection (a similar level is shown in $a$ for animal C). The left fornix (b) and right fornix $(c)$ are shown at a level caudal to the transection. The normal size of the fornix and the distribution of AChE are shown in animal $C$. There is a small patch of lower fiber staining on the right side (arrow) that resulted from direct damage to the right medial septal nucleus. Note that the fornix at the rostral level $(a)$ is substantially shrunken in cases FX1 and FX2 relative to animal $C$. Moreover, there is a marked buildup of AChE fiber staining in this proximal portion of the fornix in the lesioned animals. Fiber staining distal to the transection in these animals $(b$ and $c$ ) was completely eliminated and indicated a complete transection of the septohippocampal projection. The dark patch in the midportion of the fimbria (panel $b$ of $F X 2$ ) represents nonspecific, background labeling. Scale bar, $1 \mathrm{~mm}$.

completely transected but on the right a small bulge of laterally situated fornix was left intact. In FX5, the laterodorsal nucleus was damaged bilaterally. The medial mammillary nuclei were shrunken to approximately $68 \%$ of control values. In monkey FX6, there was slight bilateral damage to the caudal pole of the mediodorsal nucleus and bilateral damage to the ventral cingulate cortex. The medial mammillary nuclei were shrunken to approximately $45 \%$ of control values.

It is relevant to the lesions in monkeys FX5 and FX6 that the subicular projection to the diencephalon is spatially restricted in the fornix and occupies the most medial zone. Thus, it is likely that the efferent projections of the hippocampal formation to the mammillary nuclei and to the anterior thalamus were entirely transected in these cases, despite sparing of the lateral tips of the fornix on one side. It should be noted that the medial mammillary nuclei were as shrunken in these 2 cases as in the cases with complete bilateral transection (Table 1).

\section{Mammillary nuclei lesions}

The 2 monkeys in this group had rather complete damage of the medial mammillary nuclei (Fig. 4). The damage appeared to result both from thermal coagulation and mechanical disruption caused by insertion of the radio frequency probe. In both cases, there was compression damage to the entorhinal cortex on the side (left) from which the hypothalamus was approached. This damage consisted of cell loss in layers II and III of the rostral third of the entorhinal cortex. There was also some patchy cell loss in adjacent portions of area TE.

In monkey MN1 there were only a few isolated patches of what appeared to be medial mammillary neurons in the most caudal aspect of the mammillary complex (Fig. 4). These patches comprised only $4 \%$ of the normal volume of the nucleus (Table 1). Rostral to the mammillary complex, the lesion encompassed the premammillary nuclei and the ventral half of the caudal ventromedial hypothalamic nucleus. There was no direct involvement of the thalamus, but the mammillothalamic tract was heavily gliotic and the anterior thalamic nuclei appeared to be somewhat shrunken. The lesion did not extend caudally beyond the mammillary complex, and the ventral tegmental area appeared normal.

In monkey MN2, there were a few patches of what appeared to be medial mammillary neurons remaining on both sides (Fig. 

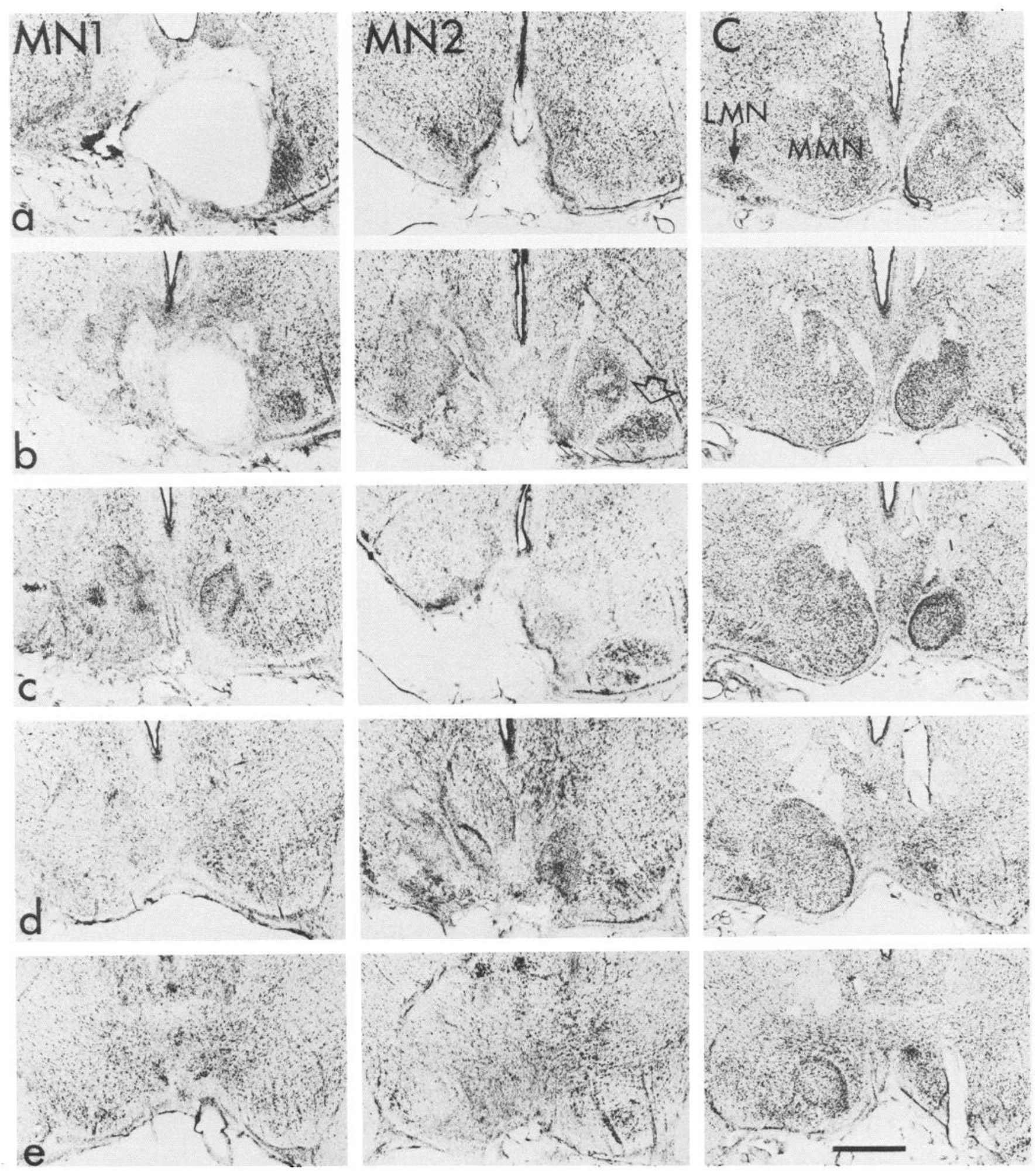

Figure 4. Photomicrographs of coronal, thionin-stained sections through the region of the mammillary complex in cases $M N 1$ and $M N 2$ in which the mammillary nuclei were largely eliminated and in case $C$ in which the lesion was located unilaterally and anterior to the right side of the mammillary complex. Sections are spaced at $250 \mu \mathrm{m}$ and are arranged from rostral $(a)$ to caudal $(e)$. Note the marked shrinkage of the right mammillary nucleus in animal C, which resulted from the transection of afferent fornix fibers. A similar, but bilateral, shrinkage was observed in animals with hippocampal and fornix lesions. In case $M N 1$, there were virtually no identifiable regions of the mammillary complex. In case $M N 2$, the medial mammillary nucleus was nearly completely eliminated, but the lateral mammillary nucleus was intact on the right side $(a r r o w, b)$. The region of the mammillary complex in both cases was markedly gliotic, as was the mammillothalamic tract. Damage to the mammillary nuclei appeared to be a consequence both of thermal coagulation and mechanical disruption consequent to the insertion of the radio frequency probe. Scale bar, $1 \mathrm{~mm}$. 

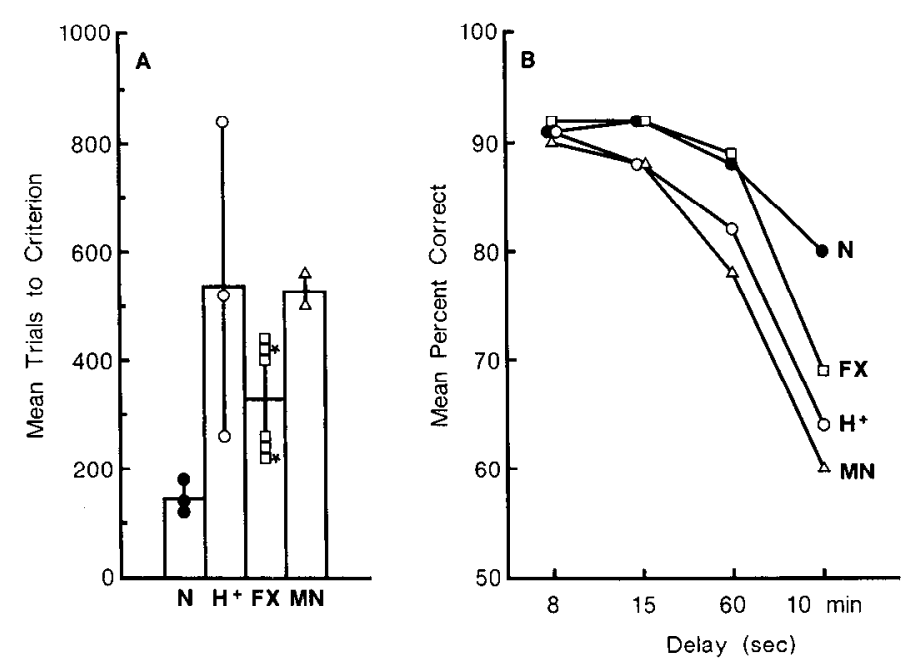

Figure 5. Performance on the delayed nonmatching to sample task by normal monkeys $(N)$ and by monkeys with lesions of the hippocampal formation $\left(H^{+}\right)$, fornix $(F X)$, or mammillary nuclei $(M N)$. $A$, Initial learning of the task with a delay of $8 \mathrm{sec}$. Symbols show trials to criterion for individual animals. $B$, Performance at delays for the same groups. The asterisk designates $F X$ monkeys operated on using a direct neurosurgical, rather than a stereotaxic, approach.

4), but these comprised less than $1 \%$ of the normal volume of this nucleus (Table 1). The lateral mammillary nucleus was intact on the right side (Fig. 4). Rostrally, the lesion slightly damaged the ventromedial hypothalamic nucleus. In addition, the surgical procedure apparently produced a bilateral thalamic infarction. At rostral levels there was massive gliosis in the reticular nucleus and in the internal capsule ventrolateral to the antcrior thalamic nuclci. At more caudal levels, a linear lesion extended along the internal medullary lamina and involved the midline thalamic nuclei and the medial aspect of the ventrobasal complex. The lesion undercut the mediodorsal nucleus on both sides but did not directly involve it. Caudal to this point the lesion was confined to the centromedian-parafascicular complex.

\section{Behavioral findings}

\section{Delayed nonmatching to sample}

The 4 groups differed in their ability to learn the basic task with a delay of $8 \sec (F[3,11]=5.6, p<0.05)$. Figure $5 A$ and Table 2 show that the normal group required a mean of only 147 trials to reach learning criterion on the basic task, while the $\mathrm{H}^{+}$group required a mean of 540 trials, the FX group required a mean of 330 trials, and the MN group required a mean of 530 trials. Compared with the normal group, all operated groups were impaired ( $p s<0.05$ ). Also, the $\mathrm{MN}$ group required more trials to learn the basic task than did the FX group $(t[7]=3.0, p<$ 0.05 ).

While all 3 operated groups were able to reach the learning criterion on the basic $(8 \mathrm{sec})$ portion of the nonmatching to sample task, further analysis revealed that the performance of the MN group was qualitatively different from that of the $\mathrm{H}^{+}$ and FX groups in at least 2 ways. First, monkeys with $\mathrm{MN}$ lesions achieved a $90 \%$ performance score (i.e., $90 \%$ or more correct during one single 20 -trial session) within the same number of sessions as did normal monkeys (mean number of sessions prior to a session of $90 \%$ correct: $\mathrm{N}=8, \mathrm{MN}=10 ; t[3]=0.20$, $p>0.10$ ). In contrast, every monkey in the $\mathrm{H}^{+}$and FX groups

\begin{tabular}{|c|c|c|c|c|c|}
\hline \multirow[b]{2}{*}{ Animal } & \multirow{2}{*}{$\begin{array}{l}\text { Trials to } \\
\text { criterion }\end{array}$} & \multicolumn{4}{|c|}{ Delays } \\
\hline & & $8 \mathrm{sec}$ & $15 \mathrm{sec}$ & $60 \mathrm{sec}$ & $10 \mathrm{~min}$ \\
\hline \multicolumn{6}{|l|}{$\mathrm{N}$} \\
\hline 1 & 120 & 91 & 98 & 91 & 82 \\
\hline 2 & 180 & 92 & 92 & 90 & 85 \\
\hline 3 & 140 & 90 & 86 & 83 & 74 \\
\hline Mean & 147 & 91 & 92 & 88 & 80 \\
\hline \multicolumn{6}{|l|}{$\mathrm{H}^{+}$} \\
\hline 1 & 260 & 91 & 85 & 78 & 60 \\
\hline 2 & 520 & 92 & 90 & 78 & 64 \\
\hline 3 & 840 & 91 & 88 & 91 & 68 \\
\hline Mean & 540 & 91 & 88 & 82 & 64 \\
\hline \multicolumn{6}{|l|}{ FX } \\
\hline 1 & 220 & 95 & 85 & 95 & 78 \\
\hline 2 & 420 & 90 & 99 & 93 & 68 \\
\hline 3 & 420 & 92 & 87 & 75 & 62 \\
\hline 4 & 240 & 90 & 95 & 95 & 83 \\
\hline 5 & 260 & 91 & 92 & 89 & 62 \\
\hline 6 & 420 & 92 & 93 & 86 & 62 \\
\hline Mean & 330 & 92 & 92 & 89 & 69 \\
\hline \multicolumn{6}{|l|}{$\mathrm{MN}$} \\
\hline 1 & 500 & 90 & 85 & 74 & 62 \\
\hline 2 & 560 & 90 & 90 & 82 & 57 \\
\hline Mean & 530 & 90 & 88 & 78 & 60 \\
\hline
\end{tabular}

$\mathrm{N}$, normal monkeys; $\mathrm{H}^{+}, \mathrm{FX}, \mathrm{MN}$, monkeys with lesions of the hippocampal formation, fornix, and mammillary nuclei, respectively.

required more sessions to achieve the $90 \%$ level of performance than any of the normal monkeys or any monkey in the MN group (mean for the $\mathrm{H}^{+}$group $=24$ sessions; mean for the FX group $=13$ sessions). Second, while monkeys in the normal, the $\mathrm{H}^{+}$, and the FX groups reached criterion without difficulty once the $90 \%$ level of performance was achieved, monkeys with $\mathrm{MN}$ lesions were unable to maintain the consistently accurate performance required to achieve learning criterion, i.e., $90 \%$ correct during 100 consecutive trials. Often, after a session of $90 \%$ correct, the performance of monkeys with MN lesions dipped to 60 or $70 \%$ correct (mean number of 20 -trial sessions required to reach learning criterion after the first session in which a $90 \%$ correct score was obtained: $\mathrm{N}=0.3, \mathrm{H}^{+}=2.3, \mathrm{FX}=1.0, \mathrm{MN}$ $=14.0$ ).

Figure $5 B$ shows performance as the delay was increased from $8 \mathrm{sec}$ to $10 \mathrm{~min}$. Table 2 shows individual scores for this task. An analysis of variance involving all 4 groups and 3 delays ( 15 $\mathrm{sec}, 60 \mathrm{sec}$, and $10 \mathrm{~min}$ ) revealed a significant effect of group $(F[3,10]=4.4, p<0.05)$, delay $(F[2,20]=61.4, p<0.001)$, and no significant group $\times$ delay interaction $(F[6,20]=1.3, p$ $>0.1)$. Separate comparisons between the normal group and each of the 3 operated groups across the same 3 delays $(15 \mathrm{sec}$, $60 \mathrm{sec}$, and $10 \mathrm{~min}$ ) revealed that the $\mathrm{H}^{+}$group fell just short of significance (average of 3 longest delays: $\mathrm{H}^{+}=78 \%, \mathrm{~N}=87 \%$, $t[4]=2.4, p=0.07$ ), and the MN group was significantly impaired $(t[3]=6.6, p=0.005)$. The FX group performed normally $(t[7]=0.9, p>0.10)$. Both the $\mathrm{H}^{+}$and the MN groups were impaired at the longest $(10 \mathrm{~min})$ delay $(t \mathrm{~s}>4.1, p \mathrm{~s}<0.05)$. Although the FX group did score numerically lower than the 


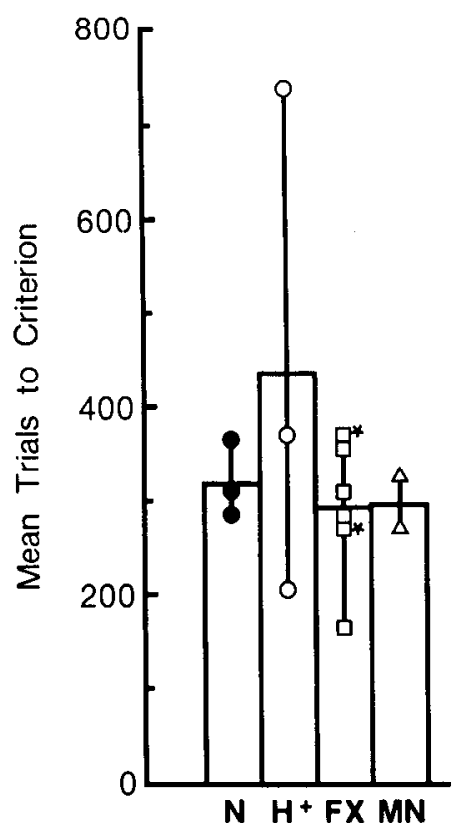

Figure 6. Average score on 2 pattern-discrimination tasks by normal monkeys $(N)$ and by monkeys with lesions of the hippocampal formation $\left(H^{+}\right)$, fornix $(F X)$, or mammillary nuclei $(M N)$. Symbols show scores for individual monkeys. The asterisk designates $F X$ monkeys operated on using a direct neurosurgical, rather than a stereotaxic, approach.

normal group at the longest $(10 \mathrm{~min})$ delay, a separate comparison involving this delay did not reach significance $(t[7]=$ $1.9, p=0.10$ ). In summary, the $\mathrm{H}^{+}$and MN groups were impaired on the delayed nonmatching to sample task, especially at the long delays. The FX group performed well but scored numerically more poorly than the control group at the longest delay.

\section{Pattern discrimination}

The 2 pattern-discrimination problems proved to be equally difficult. Accordingly, for each monkey, the number of trials required to learn the 2 problems was averaged together (Fig. 6). None of the operated groups differed from normal monkeys in terms of the number of trials required to learn the pattern discriminations (all $p \mathrm{~s}>0.1$ ). The $\mathrm{H}^{+}$monkey requiring the most trials to reach criterion was the one that had sustained bilateral damage to the optic radiations.

In a previous study (Zola-Morgan and Squire, 1984), monkeys with conjoint hippocampus-amygdala lesions also performed well on these same 2 pattern-discrimination tasks but poorly on the first few trials of each testing day. In another study, a group of 8 monkeys with lesions of the hippocampal formation (including the three $\mathrm{H}^{+}$monkeys of the present study) also performed poorly on the first few trials of these same pattern discrimination tasks (Zola-Morgan and Squire, 1986). It was suggested that this impairment, which was always observed during the first 5 trials of each testing day, reflects a component of the task that is not skill-like (see Zola-Morgan and Squire, 1984). Accordingly, in the present study we analyzed separately the scores for the first 5 trials of each test day and the scores for the remaining trials of each test day.

The 4 groups differed in their performance on the first 5 trials of each test day $(F[3,11]=52.1, p<0.001)$. The mean percentage correct during the first 5 trials of all testing sessions was

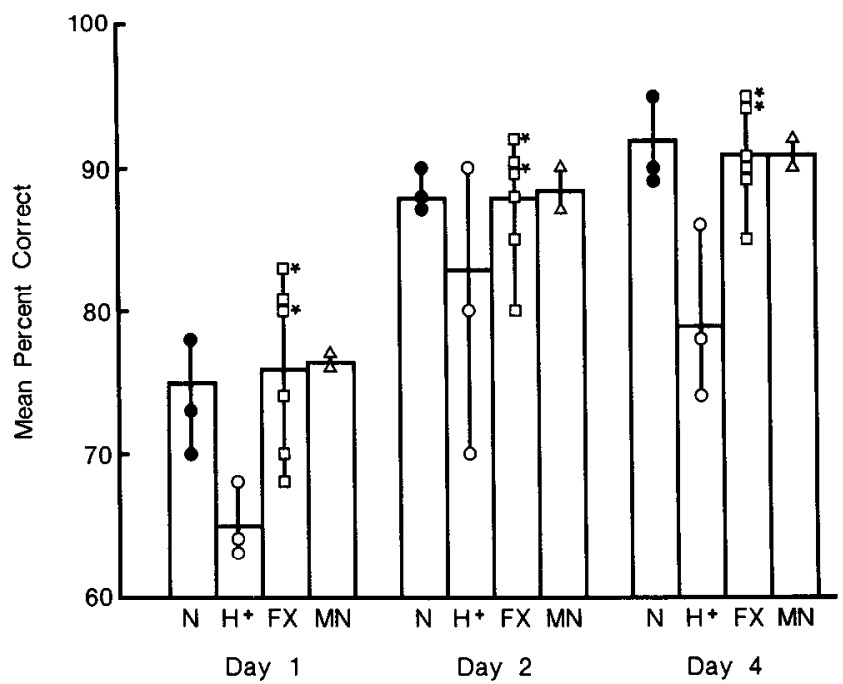

Figure 7. Average daily performance on 4 object-discrimination tasks by normal monkeys $(N)$ and by monkeys with lesions of the hippocampal formation $\left(H^{+}\right)$, fornix $(F X)$, or mammillary nuclei $(M N)$. Symbols show scores for individual monkeys. The asterisk designates $F X$ monkeys operated on using a direct neurosurgical, rather than a stereotaxic, approach.

as follows: $\mathrm{N}=77 \%, \mathrm{H}^{+}=53 \%, \mathrm{MN}=53 \%, \mathrm{FX}=72 \%$. Separate comparison between groups showed that monkeys with $\mathrm{H}^{+}$lesions and monkeys with $\mathrm{MN}$ lesions were impaired ( $\mathrm{N}$ vs $\mathbf{H}^{+}$: $t[4]=11.9, p<0.001 ; \mathrm{N}$ vs $\mathrm{MN}: t[3]=11.5, p<0.001)$. Monkeys with FX lesions performed normally (N vs FX: $t$ [7] $=1.9, p>0.10$ ). During the remaining trials of each test day, all groups performed similarly $\mathrm{N}=69 \%, \mathrm{H}^{+}=68 \%, \mathrm{MN}=$ $66 \%, \mathrm{FX}=73 \%$ ). These findings support the suggestion that performance at the beginning of each test day during pattern discrimination learning depends on a kind of memory that is sensitive to amnesia (Squire and Zola-Morgan, 1983). In summary, all the operated groups performed normally overall on the 2 pattern-discrimination tasks, but the $\mathrm{H}^{+}$and $\mathrm{MN}$ groups were impaired on the first few trials of each test day.

\section{Delayed retention of object discriminations}

The data for all 4 discriminations were averaged together, and the mean percentage correct score was calculated for each day of testing (Fig. 7). A 2-way analysis of variance involving all 4 groups revealed an overall group effect $(F[3,11]=8.8, p<$ $0.01)$, an effect across days $(F[2,22]=62.7, p<0.001)$, and no group $\times$ day interaction $(F[6,22]=0.60)$. Separate comparisons between groups, with scores averaged across the $3 \mathbf{d}$ (Table 3), showed that the monkeys with $\mathrm{H}^{+}$lesions were impaired, whereas monkeys with MN or FX lesions performed normally $\left(\mathrm{H}^{+}\right.$vs $\mathrm{N}, t[4]=3.4, p<0.05$; all other $\left.t \mathrm{~s}<0.10\right)$.

Another way of describing these data is to note that all monkeys were eventually able to achieve a run of 9 out of 10 correct trials by the end of Day 2. Normal monkeys required a median of 9 trials to learn the 4 tasks (medians are used here to permit direct comparison with the findings reported in Zola-Morgan and Squire, 1985), $\mathrm{H}^{+}$monkeys required 20 trials, FX monkeys required 8.5 trials, and $\mathrm{MN}$ monkeys required 10 trials. By this measure, the $\mathrm{H}^{+}$group performed worse than any of the other groups $(t \mathrm{~s}>2.6, p \mathrm{~s}<0.06$ ). In addition, having achieved a high level of performance by the end of Day 2, the $\mathrm{H}^{+}$animals performed worse on Day 4 than any other group ( $t \mathrm{~s}>2.9, p \mathrm{~s}$ 


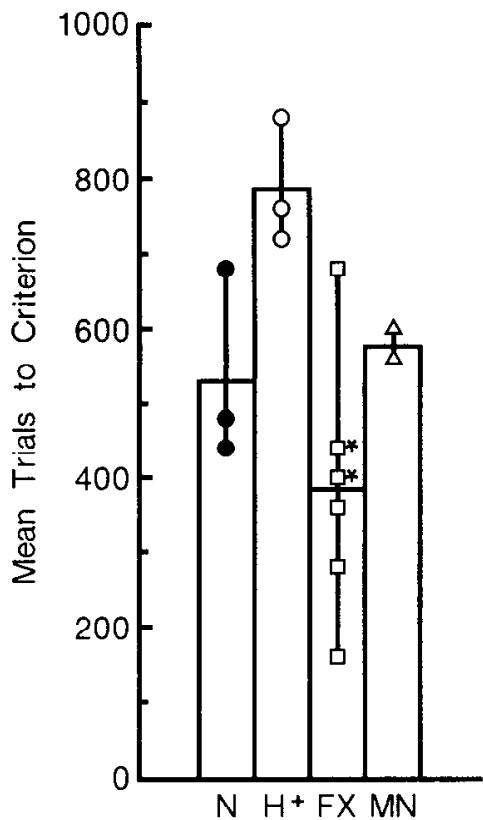

Figure 8. Performance on the 8-pair concurrent task by normal monkeys $(N)$ and by monkeys with lesions of the hippocampal formation $\left(H^{+}\right)$, fornix $(F X)$, or mammillary nuclei $(M N)$. Symbols show scores for individual monkeys. The asterisk designates $F X$ monkeys operated on using a direct neurosurgical, rather than a stereotaxic, approach.

$<0.05$ ). In this sense, the $\mathrm{H}^{+}$monkeys exhibited impaired retention on Day 4 of what had been learned on Days 1 and 2. In summary, the $\mathrm{H}^{+}$group was impaired on the object-discrimination task, but the FX and MN groups performed normally.

\section{Concurrent discrimination}

Scores for each group are presented in Figure 8. Individual scores appear in Table 3. An analysis of variance revealed a significant group effect $(F[3,11]=5.0, p<0.05)$. Further comparisons between groups showed that only the monkeys with $\mathrm{H}^{+}$lesions were impaired, whereas monkeys with $\mathrm{MN}$ or $\mathrm{FX}$ lesions performed normally (mean trials to criterion: $\mathrm{N}=533, \mathrm{H}^{+}=787$, $\mathrm{FX}=387, \mathrm{MN}=580$ ). The monkeys with $\mathrm{H}^{+}$lesions performed more poorly than any other group $(t \mathrm{~s}>2.8, p \mathrm{~s}<0.05)$. The monkeys in the other 2 operated groups performed normally $(p s>0.10)$.

\section{Delayed response with and without distraction}

The group of normal monkeys and the 3 operated groups required a similar number of trials to learn the basic task at a delay of $8 \mathrm{sec}$ (means: $\mathrm{N}=280$ trials, $\mathrm{H}^{+}=347$ trials, $\mathrm{FX}=$ 344 trials, $\mathrm{MN}=490$ trials; Fig. 9A). Note, however, that 2 of the 6 monkeys in the FX group were quite impaired at learning the basic task, and one of them was unable to reach criterion within 1000 trials. This animal was given a score of 1000 in Figure $9 A$ and was not tested on the delays.

Figure $9 B$ shows the effects of increasing the delay interval with and without a distracting event interposed during the delays. A 3-way analysis of variance [ 4 groups, 2 conditions (distraction and no distraction), and 2 delays (15 and $30 \mathrm{sec})] \mathrm{rc}-$ vealed significant effects of group $(F[3,10]=4.2, p<0.05)$, condition $(F[1,10]=54.7, p<0.001)$, and delay $(F[1,10]=$ $241.2, p<0.001)$. In addition, there was a significant interaction of group $\times$ delay $(F[3,10]=7.4, p<0.01)$ and an interaction
Table 3. Performance on tasks sensitive to amnesia

\begin{tabular}{|c|c|c|c|c|c|}
\hline Animal & $\begin{array}{l}\text { Delayed } \\
\text { non- } \\
\text { matching }\end{array}$ & $\begin{array}{l}\text { Object } \\
\text { retention }\end{array}$ & $\begin{array}{l}\text { Con- } \\
\text { current } \\
\text { discrim- } \\
\text { ination } \\
\end{array}$ & $\begin{array}{l}\text { Delayed } \\
\text { response }\end{array}$ & $\begin{array}{l}\text { De- } \\
\text { layed } \\
\text { non- } \\
\text { match- } \\
\text { ing } \\
\text { retest }\end{array}$ \\
\hline \multicolumn{6}{|l|}{$\mathrm{N}$} \\
\hline 1 & 90 & 83 & 440 & 80 & 84 \\
\hline 2 & 89 & 87 & 480 & 75 & 83 \\
\hline 3 & 81 & 84 & 680 & 64 & 85 \\
\hline Mean & 87 & 85 & 533 & 73 & 84 \\
\hline \multicolumn{6}{|l|}{$\mathbf{H}^{+}$} \\
\hline 1 & 74 & 75 & 760 & 53 & 76 \\
\hline 2 & 77 & 72 & 720 & 63 & 82 \\
\hline 3 & 82 & 80 & 880 & 62 & 84 \\
\hline Mean & 78 & 76 & 787 & 59 & 81 \\
\hline \multicolumn{6}{|l|}{ FX } \\
\hline 1 & 86 & 88 & 360 & 59 & - \\
\hline 2 & 87 & 88 & 440 & - & - \\
\hline 3 & 75 & 85 & 400 & 80 & 94 \\
\hline 4 & 91 & 84 & 160 & 78 & 100 \\
\hline 5 & 81 & 80 & 280 & 75 & 99 \\
\hline 6 & 80 & 83 & 680 & 79 & 94 \\
\hline Mean & 83 & 85 & 387 & 74 & 97 \\
\hline \multicolumn{6}{|l|}{$\mathrm{MN}$} \\
\hline 1 & 74 & 85 & 600 & 79 & 86 \\
\hline 2 & 76 & 85 & 560 & 76 & 85 \\
\hline Mean & 75 & 85 & 580 & 78 & 86 \\
\hline
\end{tabular}

The score for the delayed nonmatching task is the percentage correct score averaged across 3 delays ( $15 \mathrm{sec}, 60 \mathrm{sec}$, and $10 \mathrm{~min}$ ). The score for the object-retention task is the percentage correct score averaged across 3 test days. For concurrent discrimination, the score is the number of trials required to reach the learning criterion. The score for the delayed response task and for the retest of the delayed nonmatching task is the percentage correct score averaged across 2 delays $(15$ and $30 \mathrm{sec}$ ) and 2 distraction conditions (with and without). $\mathrm{N}$, normal monkeys; $\mathrm{H}^{+}$, monkeys with lesions of the hippocampal formation; FX, monkeys with bilateral fornix transection; MN, monkeys with circumscribed lesions of the mammillary nuclei.

of condition $\times$ dclay $(F[1,10]=6.8, p<0.05)$. These findings show that the 4 groups differed from each other, that performance on the distraction trials was worse overall than on the no-distraction trials, and that performance on $30 \mathrm{sec}$ delay trials was worse overall than performance on $15 \mathrm{sec}$ delay trials. The group $\times$ delay interaction shows that, when the delay interval was increased from 15 to $30 \mathrm{sec}$, some groups were affected more than others. The condition $\times$ delay interaction shows that the effect of distraction trials was greater at the $30 \mathrm{sec}$ delay than at the 15 sec delay.

Separate comparisons (Table 3) showed that, compared with the normal group, monkeys with $\mathrm{H}^{+}$lesions were marginally impaired overall (on the 15 and $30 \mathrm{sec}$ delays with and without distraction trials, $t[4]=2.5, p<0.07$; for the no-distraction trials alone, $t[4]=2.7, p=0.05$ ). The $\mathbf{H}^{+}$group was also significantly impaired overall (on the 15 and $30 \mathrm{sec}$ delays with and without distraction) relative to both the $\mathrm{MN}$ and FX groups $(t \mathrm{~s}>2.6, p \mathrm{~s}<0.05)$. The MN group and the FX group performed normally $(t \mathrm{~s}<0.2, p \mathrm{~s}>0.10)$. In summary, after successfully learning the delayed response task at an $8 \mathrm{sec}$ delay, the $\mathrm{H}^{+}$group was impaired when the delay was then increased 

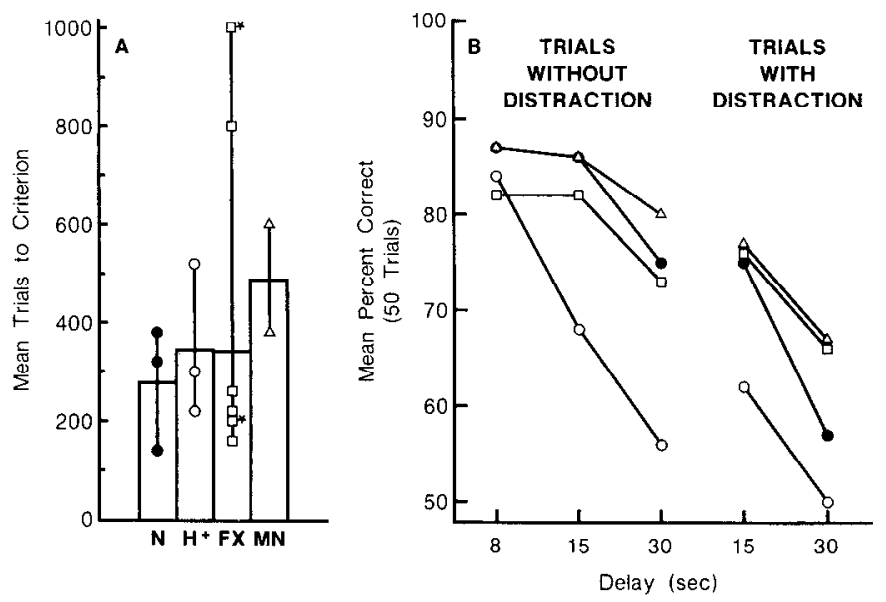

Figure 9. Delayed response performance by normal monkeys $(N)$ and by monkeys with lesions of the hippocampal formation $\left(H^{+}\right)$, fornix $(F X)$, or mammillary nuclei $(M N)$. $A$, Initial learning of the task with a delay of $8 \mathrm{sec}$. Symbols show trials to criterion for individual animals. The asterisk designates $F X$ monkeys operated on using a direct neurosurgical, rather than a stereotaxic, approach. One of these monkeys failed to reach criterion within 1000 trials and was not tested on the delays. $B$, Performance at delays by normal monkeys and by monkeys in the 3 operated groups. For half of the 15 and $30 \mathrm{sec}$ delays, a distraction task was introduced during the delay.

to 15 and $30 \mathrm{sec}$. The other operated groups performed normally.

\section{Nonmatching to sample with and without distraction}

All monkeys had experience with delayed nonmatching to sample 18-26 months previously. This task was the first one learned by each group (see Fig. 5). The second testing on this task differed in 2 ways from the first. First, a distracting event was introduced during some of the delay intervals. Second, delays of 8,15 , and $30 \mathrm{sec}$ were used instead of $8 \mathrm{sec}, 15 \mathrm{sec}, 60 \mathrm{sec}$, and $10 \mathrm{~min}$.

Figure $10 \mathrm{~A}$ shows the mean number of trials required to reach learning criterion on the basic $(8 \mathrm{sec})$ task the second time it was given $\left(\mathrm{N}=73, \mathrm{H}^{\prime}=106, \mathrm{FX}=0, \mathrm{MN}=30\right)$. Note that only 4 of the 6 monkeys in the FX group were given this test. Figure $10 B$ shows the effect of increasing the delay interval and introducing a distraction task during some of the delay intervals. A 3-way analysis of variance [ 4 groups, 2 conditions (distraction and no distraction), and 2 delays ( 15 and $30 \mathrm{sec}$ ) revealed significant effects of group $(F[3,9]=16.3, p<0.001)$, condition $(F[1,9]=5.5, p<0.05)$, and delay $(F[1,9]=9.9, p<0.01)$. None of the interaction terms approached significance. These findings show that the 4 groups differed from each other, that performance on the distraction trials was worse overall than performance on the no-distraction trials, and that performance on 30-sec delay trials was worse overall than performance on 15-sec delay trials.

Separate comparisons between groups (averaged across the 15 and $30 \mathrm{sec}$ delays with and without distraction) showed that the monkeys with FX lesions performed better than normal $(t[5]=7.3, p<0.01)$. The $\mathrm{H}^{+}$group performed numerically more poorly than the $\mathrm{MN}$ and $\mathrm{N}$ groups, but because of the consistently higher scores of monkey $\mathrm{H}^{+} 3$ on this task, these differences did not reach significance ( $p$ s $>0.10)$. In summary, the FX group performed better than normal, perhaps because of a preference for novelty previously noted in monkeys with
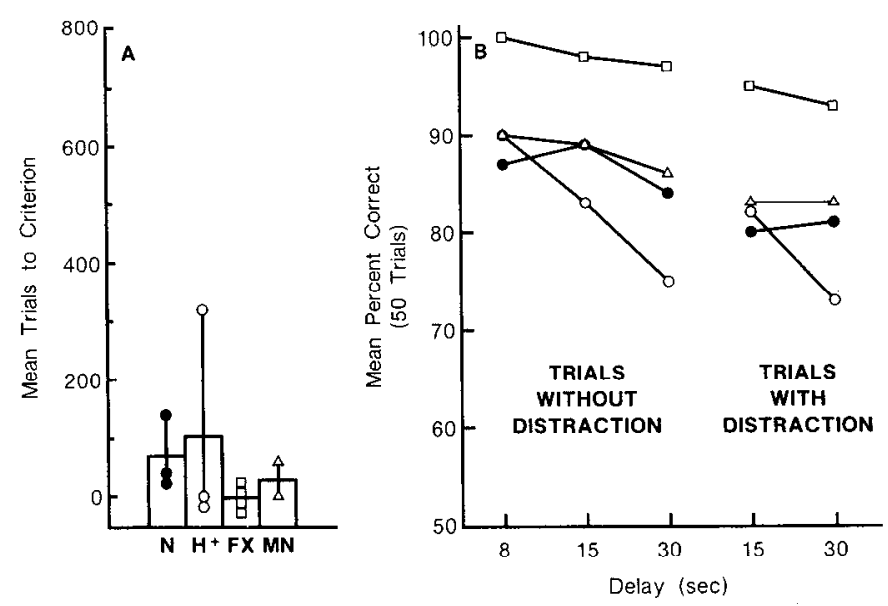

Figure 10. Delayed nonmatching to sample performance by normal monkeys $(N)$ and by monkeys with lesions of hippocampal formation $\left(H^{+}\right)$, fornix $(F X)$, or the mammillary nuclei $(M N)$. $A$, Initial learning of the task with a delay of $8 \mathrm{sec}$. Symbols show trials to criterion for individual animals. $B$, Performance at delays by normal monkeys and by monkeys in the 3 operated groups. For half of the 15 and $30 \mathrm{sec}$ delay trials, a distraction task was introduced during the delay.

fornix lesions (Zola-Morgan et al., 1983). The $\mathrm{H}^{+}$group performed numerically more poorly than the other groups, but not significantly so. It is also noteworthy that the $\mathrm{H}^{+}$group performed about the same as when the task was first given 1.5-2 years earlier.

In order to assess more thoroughly whether the memory impairment in the $\mathrm{H}^{+}$group was enduring, this group was tested on delayed nonmatching to sample on two other occasions, 42 and 53 months after surgery, using the same delays $(8 \mathrm{scc}, 15$ $\mathrm{sec}, 60 \mathrm{sec}$, and $10 \mathrm{~min}$ ) and the same procedure as when the task was first given (Fig. 11). The scores were just as poor on the final 2 tests as on initial testing [initially, the average score across the 3 delays ( $15 \mathrm{sec}, 60 \mathrm{sec}$, and $10 \mathrm{~min}$ ) was $78 \%$; at 42 months, the average score was $81 \%$; at 53 months, the average score was $79 \%$ ]. The $\mathrm{H}^{+}$group performed more poorly than the control group at the 2 long postoperative intervals (at 42 months, $p=0.07$; at 53 months, $p=0.05$ ).

\section{Lifesaver motor skill}

All groups learned the Lifesaver task at a normal rate (Fig. 12). Only 4 monkeys in the FX group were tested. A 2-way analysis of variance involving 4 groups and 8 test days revealed a significant effect of session $(F[7,63]=14.1, p<0.001)$, indicating that performance improved across test days. However, there was no effect of group and no significant group $\times$ session interaction $(F \mathrm{~s}<1.20$, ps $>0.10)$. One month after the final session of initial learning, all groups performed equivalently $(F[3,8]=0.87, p>0.10)$.

Further analyses of acquisition and retention performance by each group could not distinguish the normal monkeys from any operated group. For example, the mean number of seconds required to retrieve the Lifesaver on the first trial of each day was similar across groups $\left(\mathrm{N}=13.2 \mathrm{sec}, \mathrm{H}^{+}=11.8 \mathrm{sec}, \mathrm{FX}=10.9\right.$ sec, $\mathrm{MN}=12.7 \mathrm{sec}$ ).

\section{Discussion}

Monkeys with bilateral lesions of the hippocampal formation exhibited a long-lasting memory impairment. They were im- 


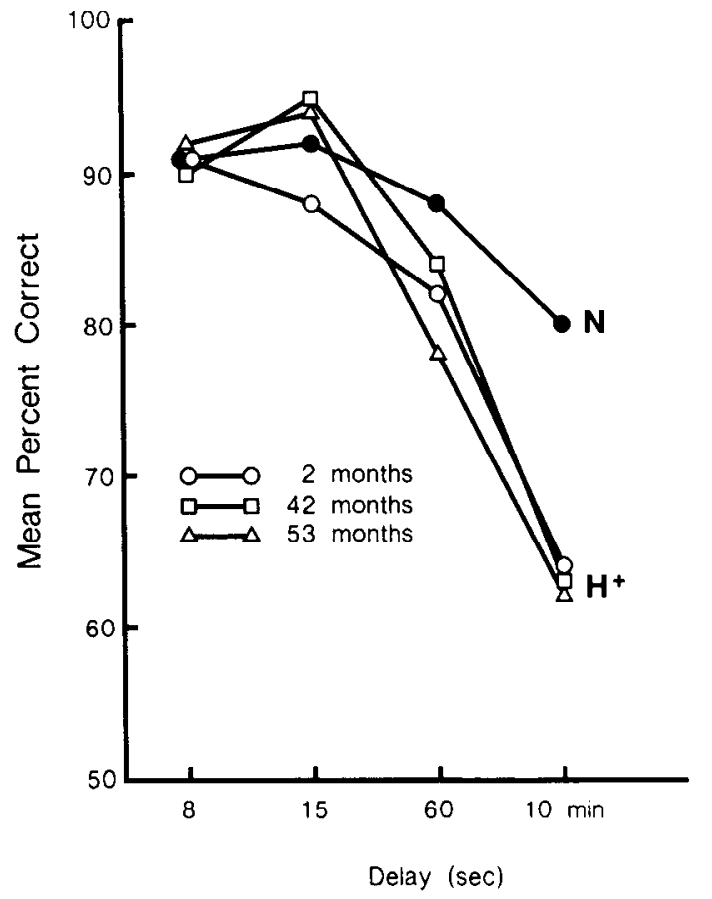

Figure 11. Delayed nonmatching to sample performance by normal monkeys $(N)$ and by monkeys with lesions of the hippocampal formation $\left(\mathrm{H}^{+}\right)$. The $\mathrm{H}^{+}$monkeys were tested on 3 different occasions: 2 months (see Fig. 4), 42 months, and 53 months after surgery.

paired on the delayed nonmatching to sample task and on 3 other memory tasks (object discrimination, concurrent discrimination, and delayed response) that were administered during the $1 \frac{112}{2}$ years after surgery. These same animals were unimpaired on pattern discrimination and motor-skill learning.

Monkeys with bilateral fornix transection were initially impaired in learning the delayed nonmatching to sample task at the 8-sec delay. After reaching criterion at the $8 \mathrm{sec}$ delay, performance was normal at the 15 and $60 \mathrm{sec}$ delays and marginally impaired at the longest $(10 \mathrm{~min})$ delay. These monkeys performed all the other tasks normally, and they performed better than normal on the delayed nonmatching to sample task when it was administered a second time 18 months after surgery. Aside from the initial difficulty with delayed nonmatching to sample, the only other sign of impairment in the fornix group was that 2 of 6 animals had difficulty achieving criterion on the delayed response task.

Monkeys with bilateral lesions of the mammillary nuclei were initially impaired on the delayed nonmatching to sample task. However, performance was normal on all the other tasks, and performance was also normal on delayed nonmatching to sample when it was re-administered 18 months after surgery. The findings for all 3 operated groups and all the tasks are summarized in Figure 13.

The impairment exhibited by monkeys with hippocampal formation lesions resembled human amnesia. The tasks that were performed poorly are ones that are also performed poorly by human amnesic patients (Oscar-Berman and Zola-Morgan, 1980; Squire et al., 1988), and the tasks that were performed well are similar to skill-like tasks that amnesic patients perform well (Brooks and Baddeley, 1976; Cohen and Squire, 1980; see also Zola-Morgan and Squire, 1984). The delayed response task may be an exception to this generalization. While monkeys with

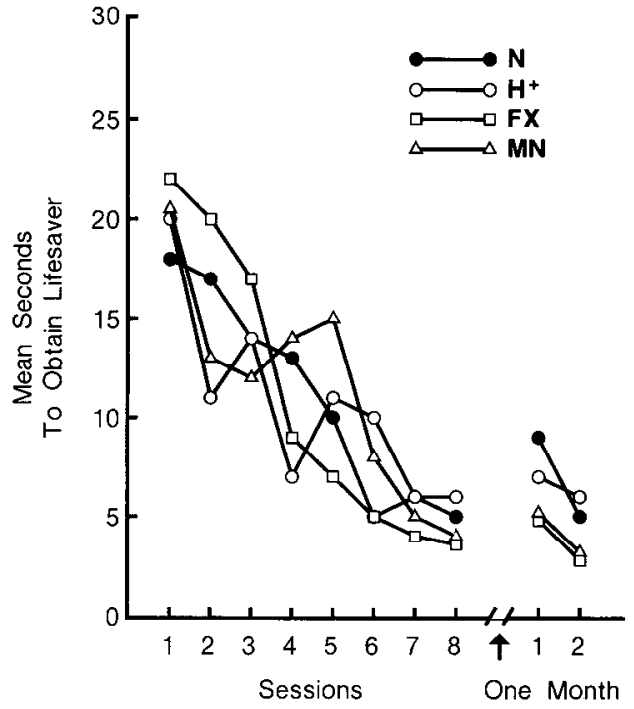

Figure 12. Acquisition of the Lifesaver motor-skill task by normal monkeys $(N)$ and by monkeys with lesions of the hippocampal function $\left(H^{\prime}\right)$, formix $(F X)$, and mammillary nuclei $(M N)$. All operated groups learned the skill at a normal rate and 1 month later retained the skill at a normal level.

hippocampal formation lesions were impaired on this task at 15- and 30-sec delays, amnesic patients performed well even at 60-sec delays (Oscar-Berman and Zola-Morgan, 1982). In the case of the delayed response task, it may be so easy for human subjects to rehearse or use other mnemonic devices during the delay interval that the task is insensitive to amnesia.

In a previous report (Zola-Morgan and Squire, 1986), we suggested that the memory impairment exhibited by monkeys with lesions of the hippocampal formation $\left(\mathrm{H}^{+}\right)$is not as severe as the impairment produced by larger lesions of the medial temporal region, i.e., a conjoint hippocampus-amygdala removal $\left(\mathrm{H}^{+} \mathrm{A}^{+}\right)$that included much of the hippocampal formation, amygdala, and adjacent cortical regions (perirhinal cortex and parahippocampal gyrus). This suggestion was based on the performance of these 2 operated groups on the delayed nonmatching to sample task (Mishkin, 1978; Zola-Morgan and Squire, 1985, 1986). In one study (Zola-Morgan and Squire, $1985), 4 \mathrm{H}^{+} \mathrm{A}^{+}$monkeys averaged $68.5 \%$ correct across the 4 delay intervals of the nonmatching to sample task; in a comparable study (Zola-Morgan and Squire, 1986), $8 \mathrm{H}^{+}$monkeys averaged $79.7 \%$ correct $\left(t[10]=5.3, p<0.001\right.$; the $3 \mathrm{H}^{+}$monkeys in the present study, who were also in the previous group of 8 monkeys, averaged $81.7 \%$ correct).

In the previous study of $\mathrm{II}^{+} \mathrm{A}^{+}$monkeys, an impairment was also observed on 3 additional tasks: retention of object discriminations, concurrent discrimination, and delayed response. The present study provides the basis for a direct comparison between the $\mathrm{H}^{+}$group and the $\mathrm{H}^{+} \mathrm{A}^{+}$group on these 3 tasks. On the object-discrimination task, $\mathrm{H}^{+}$monkeys averaged $76 \%$ correct across $3 \mathrm{~d}$ of testing, while $\mathrm{H}^{+} \mathrm{A}^{+}$monkeys averaged $70 \%$ correct $(t[5]=1.9, p>0.10)$. On the concurrent discrimination task, $\mathrm{H}^{+}$monkeys required an average of 787 trials to reach criterion, while $\mathrm{H}^{+} \mathrm{A}^{+}$monkeys required 1080 trials $(t[5]=1.3, p>0.10)$. On the delayed response task, the $\mathrm{H}^{+}$group averaged $59.3 \%$ correct (across 2 delays and 2 distraction conditions), and the $\mathrm{H}^{+} \mathrm{A}^{+}$group averaged $58.3 \%$ correct $(p>0.10)$. Thus, comparisons across 4 tasks show that the $\mathrm{H}^{+} \mathrm{A}^{+}$group performed sig- 


\begin{tabular}{|c|c|c|c|c|c|}
\hline & \multicolumn{5}{|c|}{2 Months $\longrightarrow 1.5$ Years } \\
\hline & $\begin{array}{l}\text { Delayed } \\
\text { NMTS-I }\end{array}$ & $\begin{array}{c}\text { Object } \\
\text { Retention }\end{array}$ & $\begin{array}{c}\text { 8-Pair } \\
\text { Concurrent }\end{array}$ & $\begin{array}{l}\text { Delayed } \\
\text { Response }\end{array}$ & $\begin{array}{l}\text { Delayed } \\
\text { NMTS-II }\end{array}$ \\
\hline $\mathrm{H}^{+}$ & + & + & + & + & $t$ \\
\hline $\mathrm{FX}$ & $t$ & - & - & - & - \\
\hline$M N$ & + & - & - & - & - \\
\hline
\end{tabular}

nificantly worse than the $\mathrm{H}^{+}$group only on the delayed nonmatching to sample task. Although the $\mathrm{H}^{+} \mathrm{A}^{+}$group was numerically poorer than the $\mathrm{H}^{+}$group on all 4 tasks, damage to the hippocampal formation alone can apparently produce substantial memory impairment that approaches the level of impairment associated with larger medial temporal lesions.

The present results confirm previous findings that the effects of fornix transection in the monkey are rather different from the effects of hippocampal formation lesions. In earlier studies, monkeys with fornix transection were unimpaired both on concurrent discrimination learning (Moss et al., 1981) and on delayed nonmatching to sample (Mahut et al., 1982). Monkeys with hippocampal lesions that were included in those 2 studies, as well as the monkeys with $\mathrm{H}^{+}$lesions tested in the present study, were impaired on both these tasks.

Although performance on many tasks is intact following fornix transection in the monkey, impairments do occur on tasks that require the learning of associations between stimuli and spatially directed movements (Gaffan and Harrison, 1984; Rupniak and Gaffan, 1987; Murray et al., 1989). For example, fornix transection impaired performance on a spatial delayed nonmatching to sample task (Murray et al., 1989); and fornix transection impaired performance on a conditional discrimination task in which monkeys learned to choose the object on the left when one pair of identical objects was presented and to choose the object on the right when a second pair of identical objects was presented (Gaffan and Harrison, 1984). Fornix transection did not impair the learning of object-reward associations (Gaffan et al., 1984), and fornix transection did not impair performance on a conditional discrimination task in which spatial cues indicated which one of 2 different objects was rewarded (Murray et al., 1989). The results of these studies and the present findings are consistent in suggesting that fornix transection in the monkey produces a narrower deficit than is characteristic of human amnesia.

The findings following lesions of the mammillary nuclei are consistent with previous reports that monkeys with mammillary nuclei lesions are impaired on the delayed nonmatching to sample task during the first few months after surgery (Saunders, 1983; Aggleton and Mishkin, 1985). The severity of the impairment appears to depend on whether monkeys are first given preoperative training or whether training begins postoperatively. Monkeys in the present study, which were given only postoperative training, initially exhibited a moderately severe impairment on the delayed nonmatching to sample task. In contrast, when monkeys were first given preoperative training and then tested postoperatively, only a mild impairment was observed (Aggleton and Mishkin, 1985). Importantly, whatever level of performance is observed initially, the present results suggest that performance eventually recovers to normal levels. Thus, lesions of the mammillary nuclei do not produce a long-lasting global memory impairment. Indeed, the finding that both mammillary

\begin{tabular}{|c|c|}
\hline $\begin{array}{c}\text { Pattern } \\
\text { Discrimination }\end{array}$ & $\begin{array}{c}\text { Motor } \\
\text { Skill }\end{array}$ \\
\hline- & - \\
\hline- & - \\
\hline- & - \\
\hline
\end{tabular}

Figure 13. Performance of monkeys with lesions of the hippocampal formation $\left(H^{+}\right)$, fornix $(F X)$, or the mammillary nuclei $(M N)$ on six different tasks. +, Designates impairment; normal performance; $\boxplus$, a trend toward impairment that did not reach statistical significance.

nuclei lesions and fornix transection caused only a transient memory impairment raises some uncertainty about how to interpret reports of other kinds of cognitive impairment following these lesions in the monkey. Although some examples of behavioral impairment following mammillary nuclei lesions or fornix transection are known to persist more than a year (Mahut et al., 1982; Holmes et al., 1983; Alvarez-Royo et al., 1988), most reports are based on observations limited to the first few months after surgery.

The observation that damage to the hippocampal formation, but not the fornix or the mammillary nuclei, produced persistent and global memory impairment is consistent with current understanding of the anatomical connections of these regions. The hippocampus, fornix, and mammillary nuclei have often been taken as components of a single functional circuit, with the expectation that damage at any point in the circuit should have similar effects. The subiculum of the hippocampal formation does originate a substantial projection through the fornix, and the mammillary nuclei are an important diencephalic target of this projection. However, the major efferent projection of the hippocampal formation in the monkey is directed not through the fornix but caudally to the entorhinal cortex. Moreover, the entorhinal cortex provides the major route by which the hippocampal formation exchanges information with the neocortex. Thus, damage to the fornix and mammillary nuclei need not impair information processing in the same way as direct damage to the hippocampus.

The fact that memory was intact on several tasks following bilateral fornix transection in monkeys may seem at odds with previous reports that learning and memory are profoundly impaired by bilateral fimbria-fornix lesions in rodents (Olton et al., 1979; Jarrard, 1980; Gage et al., 1983). One possibility is that fimbria-fornix lesions in the rat more severely and globally impair memory performance than the same lesion in monkeys. First, the monkey hippocampal formation receives a substantially greater direct cortical input than does the rat (Insausti et al., 1987) and, in turn, originates a more prominent projection back to neocortex (Van Hoesen, 1982). Thus, the fornix may be a relatively less important conduit of hippocampal afferent and efferent projections in the monkey than in the rat. As a result, whereas fornix transection in the monkey does not mimic the effects of hippocampal formation lesions, damage to the fornix in the rat might exert effects rather similar to the effects of hippocampal damage.

Second, in rodents, $90 \%$ of the cholinergic innervation of the hippocampal formation arrives via the fornix (Gage et al., 1983), and transection of this fiber bundle in rodents produces a nearly complete cholinergic deafferentation of the hippocampus (Milner and Amaral, 1984; Gage and Bjorklund, 1986). In the monkey, the fornix carries much less of the cholinergic input, and a more significant ventral cholinergic pathway (through the amygdala and external capsule) is observed (D. G. Amaral, unpub- 
lished observations). These considerations suggest that, if cholinergic innervation is important to the normal function of the hippocampal formation and to the performance of memory tasks, then (when comparable tasks are given) more substantial disruption of performance should occur following a bilateral fimbria-fornix lesion in the rat than following a bilateral fornix transection in the monkey. Interestingly, it was reported recently that rats were more impaired than monkeys in delayed nonmatching to sample for spatial location, which was tested in parallel in the 2 species (Markowska et al., 1989; Murray et al., 1989). Specifically, having learned the task preoperatively, monkeys with fornix transection relearned the task, albeit more slowly than normal. Rats with fimbria-fornix lesions could not relearn the task.

In conclusion, damage to the hippocampal formation produced a long-lasting memory impairment. This finding is consistent with the report that damage limited to the human hippocampus is associated with a clinically significant and enduring amncsia (Zola-Morgan ct al., 1986). Damagc to the mammillary nuclei or fornix transection produced only transient memory impairment. It is therefore unlikely that damage limited to either of these structures alone can be the cause of severe or permanent memory impairment in humans.

\section{Appendix}

According to Haubrich's Medical Meanings. A Glossary of Word Origins (Harcourt, Brace, Jovanovich, San Diego, 1984), the derivation of the term "mammillary" is from "mammillation" (a derivation of mamma, the Latin and Greek word for breast), which refers to a small excresence that bears a fancied resemblance to a little breast. The spelling of "mammillary," however, has not been commonly agreed upon (see, for example, the reference section of the present paper). The Nomina Anatomica, 5 th edition (Williams \& Wilkins, Baltimore, 1983) prepared by the International Anatomical Nomenclature Committee of the Eleventh International Congress of Anatomists (Mexico City, 1980 ) in order to standardize anatomical nomenclature, gives the spelling as "mamillary." However, the Medical Subject Headings, Annotated Alphabetical List (National Library of Medicine, Bethesda, 1987), the principal resource for identifying search-terms for computer-based searches from the National Library of Medicine (e.g., Medlars, Medline, etc.), lists only the term "mammillary." Moreover, in a survey of 7 dictionaries of medicine and biology (other than the Nomina Anatomica) available at the U.C.S.D. School of Medicine's Biomedical Library, 5 used "mammillary." In the present paper we therefore use the form "mammillary," a spelling consistent with the reported derivation of the term and with the majority of our reference sources.

\section{References}

Aggleton, J. P., and M. Mishkin (1983a) Visual recognition impairment following medial thalamic lesions in monkeys. Neuropsychologia 21: 187-197.

Aggleton, J. P., and M. Mishkin (1983b) Memory impairments following restricted medial thalamic lesions in monkeys. Exp. Brain Res. 52: 199-209.

Aggleton, J. P., and M. Mishkin (1985) Mammillary-body lesions and visual recognition in the monkey. Exp. Brain Res. 58: 190-197.

Alvarez-Royo, P., M. Mesches, J. Allen, W. Saltzmann, L. R. Squire, and S. Zola-Morgan (1988) Independence of memory functions and emotional behavior: Separate contributions of the hippocampal formation and the amygdala. Soc. Neurosci. Abstr. 14: 1043.
Bachevalier, J., R. Saunders, and M. Mishkin (1985) Visual recognition in monkeys: Effects of transection of fornix. Exp. Brain Res. 57: 547-553.

Barbizet, J. (1970) Human Memory and Its Pathology, Freeman, San Francisco.

Bonin, G., and P. Bailey (1947) The Neocortex of Macaca Mulatta, University of Illinois Press, Urbana, IL.

Brierley, J. B. (1977) Neuropathology of amnesic states. In Amnesia, C. W. M. Whitty and O. L. Zangwill, eds., pp. 199-223, Butterworths, London.

Brooks, D. N., and A. Baddeley (1976) What can amnesic patients learn? Neuropsychologia 14: 111-112.

Butters, N. (1984) Alcoholic Korsakoff's syndrome: An update. Semin Neurol. 4: 226-244.

Cohen, N. J., and L. R. Squire (1980) Preserved learning and retention of pattern analyzing skill in amnesia: Dissociation of knowing how and knowing that. Science 210: 207-209.

Damasio, A. R. (1984) The anatomic basis of memory disorders Semin Neurol. 4: 223-225.

Davis, R. T., A. A. McDowell, C. W. Deter, and J. P. Steele (1956) Performance of rhesus monkeys on selected laboratory tasks presented before and after a large single dose of whole body $\mathrm{x}$-radiation. J. Comp. Physiol. Psychol. 49: 20-26.

Gaffan, D. (1974) Recognition impaired and association intact in the memory of monkeys after transection of the fornix. J. Comp. Physiol. Psychol. 86: 1100-1109.

Gaffan, D., and S. Harrison (1984) Reversal learning by fornix-transected monkeys. Q. J. Exp. Psychol 36b: 223-234.

Gaffan, D., R. C. Saunders, E. A. Gaffan, S. Harrison, C. Shields, and M. J. Owen (1984) Effects of fornix transection upon associative memory in monkeys: Role of the hippocampus in learned action. $Q$. J. Exp. Psychol. 36b: 173-221.

Gage, F. H., and A. Bjorklund (1986) Compensatory collateral sprouting of aminergic systems in the hippocampal formation following partial deafferentation. In The Hippocampus, Vol. 3, R. G. Isaacson and K. H. Pribram, eds., pp. 33-63, Plenum, New York.

Gage, F. H., A. Bjorklund, and U. Stenevi (1983) Reinnervation of the partially deafferented hippocampus by compensatory collateral sprouting from spared cholinergic and nonadrenergic afferents. Brain Res. 268: 27-37.

Garcia-Bengochea, B., and W. A. Friedman (1987) Persistent memory loss following sections of the anterior fornix in humans. A historical review. Surg. Neurol. 27: 361-364.

Gellerman, L. W. (1933) Chance orders of alternating stimuli in visual discrimination experiments. J. Gen. Psychol. 42: 207-208.

Gudden, H. (1896) Klinische und anatomische Beitrage zur Kenntniss der multiplen Alkoholneuritis nebst Bemerkungen uber die Regenerationsvorgange im peripheren Nervensystem. Arch Psychiatr. Nerven-krank-heiten 28: 643-741.

Harlow, H. F., and J. A. Bromer (1938) A test-apparatus for monkeys. Psychol. Rec. 19: 434-438.

Hartley, L. H., R. Roger, R. J. Nicolosi, and T. Hartley (1984) Bloodpressure values in Macaca fascicularis. J. Med. Primatol. 13: 183189.

Hassler, R., and T. Riechert (1957) Ueber einen Fall von doppelseitiger Forniocotomie bei sogenannter temporaler Epilepsie. Acta Neurochir. (Wien) 5: 330-340.

Heilman, K. M., and G. W. Seipert (1977) Korsakoff's syndrome resulting from bilateral fornix lesions. Neurology 27: 490-493.

Holmes, E. J., N. Butters, S. Jacobson, and B. M. Stein (1983) An examination of the effects of mammillary-body lesions on reversal learning sets in monkeys. Physiol. Psychol. 11:159-165.

Insausti, R., D. G. Amaral, and W. M. Cowan (1987) The entorhinal cortex of the monkey: II. Cortical afferents. J. Comp. Neurol. 264: $356-395$

Jarrard, L. E. (1980) Selective hippocampal lesions and behavior. Physiol. Psychol. 8: 198-206.

Mahut, H. (1972) A selective spatial deficit in monkeys after transection of the fornix. Neuropsychologia 10:65-74.

Mahut, H., and M. Moss (1984) Consolidation of memory: The hippocampus revisited. In Neuropsychology of Memory, L. R. Squire and N. Butters, eds., Guilford, New York.

Mahut, M., S. Zola-Morgan, and M. Moss (1982) Hippocampal resections impair associative learning and recognition memory in the monkey. J. Neurosci. $1: 227-240$. 
Mair, W. G. P., E. K. Warrington, and L. Weiskrantz (1979) Memory disorder in Korsakoff psychosis. A neuropathological and neuropsychological investigation of two cases. Brain 102: 749-783.

Markowitsch, H. J. (1982) Thalamic mediodorsal nucleus and memory: A critical evaluation of studies in animals and man. Neurosci. Biobeh. Rev. 6: 351-380.

Markowska, A. L., D. S. Olton, E. A. Murray, and D. Gaffan (1989) A comparative analysis of the role of fornix and cingulate cortex in memory: Rats. Exp. Brain Res. (in press).

Milner, T. A., and D. G. Amaral (1984) Evidence for a ventral septal projection to the hippocampal formation of the rat. Exp. Brain Res. 55: 579-585.

Mishkin, M. (1978) Memory in monkeys severely impaired by combined but not separate removal of amygdala and hippocampus. $\mathrm{Na}$ ture 273: 297-298.

Mishkin, M. (1982) A memory system in the monkey. Phil. R. Soc. Lond. [Biol.] 298: 85-95.

Moss, M., H. Mahut, and S. Zola-Morgan (1981) Concurrent discrimination learning of monkeys after hippocampal, entorhinal, or fornix lesions. Neurosci. 1: 227-240.

Murray, E. A., and M. Mishkin (1984) Severe tactual as well as memory deficits following combined removal of the amygdala and hippocampus in monkeys. J. Neurosci. 4; 2565-2580.

Murray, E. A., M. Davidson, D. Gaffan, D. S. Olton and S. J. Suomi (1989) Effects of fornix transection and cingulate cortical ablation on spatial memory in rhesus monkeys. Exp. Brain Res. (in press).

Olton, D. S., J. T. Becker, and G. E. Handelmann (1979) Hippocampus, space and memory. Behav. Brain Sci. 2: 313-365.

Oscar-Berman, M., and S. Zola-Morgan (1980) Comparative neuropsychology and Korsakoff's syndrome: II. Two-choice visual discrimination learning. Neuropsychologia 18: 513-525.

Oscar-Berman, M., and S. Zola-Morgan (1982) Comparative neuropsychology and Korsakoff's syndrome: III. Delayed response, delayed alternation, and DRL performance. Neuropsychologia 20: 189-202.

Papez, J. W. (1937) A proposed mechanism of emotion. Arch. Neurol. Psychiatry $38: 725-743$.

Ringo, J. I. (1988) Seemingly discrepant data from hippocampectomized macaques are reconciled by detectability analysis. Behav. Neurosci. 102: 173-177.

Rupniak, N. M. J., and D. Gaffan (1987) Monkey hippocampus and learning about spatially directed movements. J. Neurosci. 7: 23312337.

Saunders, R. C. (1983) Impairment in recognition memory after mammillary body lesions in monkeys. Soc. Neurosci. Abstr. 9: 28.
Squire, L. R. (1987) Memory and Brain, Oxford U. P., New York.

Squire, L. R., and S. Zola-Morgan (1983) The neurology of memory: The case for correspondence between the findings for man and nonhuman primates. In The Physiological Basis of Memory, 2nd ed., J. A. Deutsch, ed., pp. 199-268, Academic, New York.

Squire, L. R., S. Zola-Morgan, and K. Chen (1988) Human amnesia and animal models of amnesia: Performance of amnesic patients on tests designed for the monkey. Behav. Neurosci. 102: 210-221.

Sweet, W. H., G. A. Talland, and F. R. Ervin (1959) Loss of recent memory following section of the fornix. Trans. Am. Neurol. Assoc. 84: 876-882.

Szabo, J., and W. M. Cowan (1984) A stereotaxic atlas of the brain of the cynomolgus monkey (Macaca fascicularis). J. Comp. Neurol. 222: 265-300.

Van IIoesen, G. W. (1982) The parahippocampal gyrus. Trends Neurosci. 5: 345-350.

Victor, M., R. D. Adams, and G. H. Collins (1971) The WernickeKorsakoff Syndrome, Davis, Philadelphia.

von Bechterev, W. V. (1900) Demonstration eines Gehirns mit Zerstorung der vorderen und inneren Theile dere Hirnrinde beidere Schlaferlappen. Neuro. Zentpalbl. 19: 900-911.

Woolsey, R. M., and J. S. Nelson (1975) Asymptomatic destruction of the fornix in man. Arch. Neurol. 32: 566-568.

Zola-Morgan, S., and L. R. Squire (1984) Preserved learning in monkeys with medial temporal lesions: Sparing of motor and cognitive skills. J. Neurosci. 4: 1072-1085.

Zola-Morgan, S., and L. R. Squire (1985) Medial temporal lesions in monkeys impair memory on a variety of tasks sensitive to human amnesia. Behav. Neurosci. 9: 22-34.

Zola-Morgan, S., and L. R. Squire (1986) Memory impairment in monkeys following lesions limited to the hippocampus. Behav. Neurosci. 100: 155-160.

Zola-Morgan, S., L. R. Squire, and M. Mishkin (1982) The neuroanatomy of amnesia: The amygdala-hippocampus vs. temporal stem. Science 218: 1337-1339.

Zola-Morgan, S., J. Dabrowska, M. Moss, and H. Mahut (1983) Enhanced preference for perceptual change in the monkey after sections of the fornix, but not after ablations of the hippocampus. Neuropsychologia 21: 433-454.

Zola-Morgan, S., L. R. Squire, and D. G. Amaral (1986) Human amnesia and the medial temporal region: Enduring memory impairment following a bilateral lesion limited to field CA1 of the hippocampus. J. Neurosci. 6: 2950-2967. 